\title{
WHAT CAN INFRARED SPECTRA TELL US ABOUT THE CRYSTALLINITY OF NANO-SIZED INTERSTELLAR SILICATE DUST GRAINS?
}

\section{Supporting Information}

Lorenzo Zamirri, ${ }^{1,}$ Antoni Macià Escatllar, ${ }^{2,}$ Joan Mariñoso Guiu, ${ }^{2}$ Piero Ugliengo, ${ }^{1, *}$ and Stefan T. Bromley. ${ }^{2,3, *}$

${ }^{1}$ Dipartimento di Chimica and Nanostructured Interfaces and Surfaces (NIS) Centre, Università degli Studi di Torino, via P. Giuria 7, IT-10125, Torino, Italy.

${ }^{2}$ Departament de Ciència de Materials i Química Física and Institut de Química Teòrica i Computacional (IQTCUB), Universitat de Barcelona, C/Martí i Franquès 1, ES-08028, Barcelona, Spain.

${ }^{3}$ Institució Catalana de Recerca i Estudis Avançats (ICREA), ES-08010, Barcelona, Spain.

$\S$ Authors contributed equally to this work.

*Corresponding authors: s.bromley@ub.edu, piero.ugliengo@unito.it.

This SI file is organized into sections. S1: description of computational details. S2: technical details about the procedure we followed to generate bulk cut and amorphized NPs. S3: details of the calculations used to derive a realistic value of the full width at half maximum for the IR spectra. S4: plots of the harmonic IR spectra for all NPs. S5: Si-O bond distance and O-Si-O angle distributions for the 116 formulaunits BC, A and N NPs.

\section{S1 COMPUTATIONAL DETAILS}

In CRYSTAL, the multi-electronic wave function is constructed as an anti-symmetrized product (Slater determinant) of mono-electronic orbitals that are linear combinations of local functions centered on each atom of the crystal, known as Atomic Orbitals (AOs). In turn, AOs are expanded as linear combinations of different Gaussian functions, namely the basis set (BS) functions. The details of the BS adopted in this work (OlBS) are reported in Table S1, in Gaussian-like format.

Table S1 Detail of the basis set (in Gaussian-like format) adopted for all CRYSTAL calculations.

\begin{tabular}{rrrrrr}
\hline $\mathrm{O}$ & \multicolumn{1}{c}{0} & & & & \\
\hline & $\mathrm{S}$ & 6 & & & 1.00 \\
& & 5484.671660 & 0.0018311 & \\
& & 825.234946 & 0.0139502 & \\
& & 188.046958 & 0.0684451 & \\
& & 52.964500 & 0.2327143 & \\
& & 16.897570 & 0.4701929 & \\
& & 5.799635 & 0.3585209 & 1.00
\end{tabular}




\begin{tabular}{|c|c|c|c|c|c|}
\hline & & & 15.539616 & -0.1107775 & 0.0708743 \\
\hline & & & 3.599934 & -0.1480263 & 0.3397528 \\
\hline & & & 1.013762 & 1.1307670 & 0.7271586 \\
\hline & SP & 1 & & & 1.00 \\
\hline & & & 0.274200 & 1.0000000 & 1.0000000 \\
\hline & $\mathrm{D}$ & 1 & & & 1.00 \\
\hline & & & 0.538000 & 1.0000000 & \\
\hline $\mathrm{Mg}$ & 0 & & & & \\
\hline s & $S$ & 8 & & & 1.00 \\
\hline & & & 68371.87500 & 0.0002226 & \\
\hline & & & 9699.34009 & 0.0018982 & \\
\hline & & & 2041.17679 & 0.0110451 & \\
\hline & & & 529.86291 & 0.0500627 & \\
\hline & & & 159.18600 & 0.1691230 & \\
\hline & & & 54.68480 & 0.3670310 & \\
\hline & & & 21.23570 & 0.4004100 & \\
\hline & & & 8.74604 & 0.1498700 & \\
\hline & SP & 6 & & & 1.00 \\
\hline & & & 156.79500 & -0.0062400 & 0.0077200 \\
\hline & & & 31.03390 & -0.0788200 & 0.0642700 \\
\hline & & & 9.64530 & -0.0799200 & 0.2104000 \\
\hline & & & 3.71090 & 0.2906300 & 0.3431400 \\
\hline & & & 1.61164 & 0.5716400 & 0.3735000 \\
\hline & & & 0.64294 & 0.3066400 & 0.2328600 \\
\hline & SP & 1 & & & \\
\hline & & & 0.40000 & 1.0000000 & 1.0000000 \\
\hline $\mathrm{Si}$ & 0 & & & & \\
\hline S & S & 6 & & & 1.00 \\
\hline & & & 16120.00000 & 0.0019590 & \\
\hline & & & 2426.00000 & 0.0149300 & \\
\hline & & & 553.90000 & 0.0728500 & \\
\hline & & & 156.30000 & 0.2461000 & \\
\hline & & & 50.07000 & 0.4859000 & \\
\hline & & & 17.02000 & 0.3250000 & \\
\hline & SP & 6 & & & 1.00 \\
\hline & & & 292.700000 & -0.0027810 & 0.0044380 \\
\hline & & & 69.870000 & -0.0357100 & 0.0326700 \\
\hline & & & 22.340000 & -0.1150000 & 0.1347000 \\
\hline & & & 8.150000 & 0.0935600 & 0.3287000 \\
\hline & & & 3.135000 & 0.6030000 & 0.4496000 \\
\hline & & & 1.225000 & 0.4190000 & 0.2614000 \\
\hline & SP & 2 & & & 1.00 \\
\hline & & & 1.079000 & -0.3761000 & 0.0671000 \\
\hline & & & 0.302400 & 1.2520000 & 0.9569000 \\
\hline & SP & 1 & & & \\
\hline & & & 0.302400 & 1.2520000 & 0.9569000 \\
\hline & SP & 1 & & & \\
\hline & & & 0.170000 & 1.0000000 & 1.0000000 \\
\hline & & & & & \\
\hline
\end{tabular}


D $\quad 1$

In geometry optimizations, the thresholds controlling the accuracy of the calculation - i.e., maximum components of the gradient and displacement vectors, root-mean-square-deviations of the components of the gradient and displacement vectors, and difference in the total energy between two subsequent steps of the self-consisted-field $(\mathrm{SCF})$ procedure - were kept constant to default values $\left(4.50 \times 10^{-4}, 1.80 \times 10^{-3}, 3.00 \times 10^{-4}\right.$, $1.20 \times 10^{-4}$ and $10^{-8}$ atomic units, respectively).

In order to ensure a good accuracy in the numerical evaluation of the electron density, we used a pruned (75, 974) grid, consisting of 75 radial points and a maximum number of 974 angular points. The energetic thresholds controlling the accuracy in the evaluation of the Coulomb and exchange integrals at these points are equal to $10^{-7}, 10^{-7}, 10^{-7}, 10^{-7}$ and $10^{-25}$ hartree, respectively.

Infrared (IR) vibrational frequencies have been computed by diagonalizing the Hessian matrix of second derivatives of the total energy with respect to the atomic displacements from equilibrium positions. The Hessian elements have been numerically evaluated using the 3-points formula, which accounts for three displacements of $0.003 \AA$ of each atom along $x$-, $y$ - and $z$-axis, respectively, from the equilibrium geometry. The threshold on the total SCF energy is equal to $10^{-10}$ hartree. The absolute IR intensities have been computed according to the Barry phase method. ${ }^{1}$

Table S2 Experimental (Ref. 2) and simulated (at PBE/OlBs and Walker FF) modules (in $\AA$ ) of forsterite unit cell vectors. In brackets, the percentage errors with respect to experimental values.

\begin{tabular}{lrrr}
\hline & a (\% err) & b (\% err) & c (\% err) \\
\hline Experimental & $4.7490(-)$ & $10.1985(-)$ & $5.9792(-)$ \\
PBE/OlBS & $4.7375(-0.2)$ & $10.0742(-1.2)$ & $5.9203(-1.0)$ \\
Walker FF & $4.7892(0.9)$ & $10.2539(0.6)$ & $6.0092(0.5)$ \\
\hline
\end{tabular}


Table S3 Crystalline forsterite IR frequencies $\left(\mathrm{in}^{-1}\right)$. Comparison of our simulated data with experimental ones. $\Delta 1$ and $\Delta 2$ represent the absolute differences with respect to experimental values of IR frequencies computed at PBE/OlBS and Walker FF levels of theory.

\begin{tabular}{|c|c|c|c|c|c|}
\hline Lattice vector & Experimental* & $\mathrm{PBE} / \mathrm{OlBs}$ & $\Delta 1$ & Walker FF & $\Delta 2$ \\
\hline \multirow[t]{13}{*}{$\mathrm{a}$} & 202.6 & 239.8 & 37.2 & 193.9 & 8.7 \\
\hline & 276.7 & 294.2 & 17.5 & 293.9 & 17.2 \\
\hline & 296.0 & 326.8 & 30.8 & 308.1 & 12.1 \\
\hline & 321.1 & 357.4 & 36.3 & 349.5 & 28.4 \\
\hline & 383.2 & 445.7 & 62.5 & 378.5 & 4.7 \\
\hline & 405.2 & 455.5 & 50.3 & 438.2 & 33.0 \\
\hline & - & 515.7 & - & 483.7 & - \\
\hline & 504.8 & 543.5 & 38.7 & 520.4 & 15.6 \\
\hline & - & 583.3 & - & 574.9 & - \\
\hline & 606.1 & 630.7 & 24.6 & 657.3 & 51.2 \\
\hline & 841.3 & 916.1 & 74.8 & 812.6 & 28.7 \\
\hline & 959.9 & 956.6 & 3.3 & 960.5 & 0.6 \\
\hline & 978.2 & 974.7 & 3.5 & 995.2 & 17.0 \\
\hline \multirow[t]{13}{*}{$\mathrm{b}$} & 144.8 & 159.6 & 14.8 & 146.4 & 1.6 \\
\hline & 278.7 & 306.4 & 27.7 & 266.6 & 12.1 \\
\hline & 290.9 & 340.5 & 49.6 & 299.5 & 8.6 \\
\hline & 352.0 & 404.2 & 52.2 & 356.6 & 4.6 \\
\hline & 397.1 & 444.3 & 47.2 & 432.5 & 35.4 \\
\hline & 419.9 & 483.6 & 63.7 & 449.7 & 29.8 \\
\hline & 458.0 & 517.6 & 59.6 & 488.9 & 30.9 \\
\hline & 507.6 & 527.0 & 19.4 & 539.2 & 31.6 \\
\hline & 528.8 & 584.1 & 55.3 & 558.4 & 29.6 \\
\hline & - & 664.0 & - & 671.2 & - \\
\hline & 838.7 & 816.8 & 21.9 & 815.3 & 23.4 \\
\hline & 873.2 & 859.2 & 14.0 & 873.9 & 0.7 \\
\hline & 986.5 & 976.2 & 10.3 & 996.1 & 9.6 \\
\hline \multirow[t]{9}{*}{$\mathrm{c}$} & - & 222.9 & - & 177.3 & - \\
\hline & 278.3 & 309.2 & 30.9 & 251.2 & 27.1 \\
\hline & 292.7 & 317.9 & 25.2 & 314.2 & 21.5 \\
\hline & 306.3 & 380.7 & 74.4 & 348.1 & 41.8 \\
\hline & 411.6 & 458.5 & 46.9 & 376.5 & 35.1 \\
\hline & 418.8 & 490.9 & 72.1 & 451.9 & 33.1 \\
\hline & 478.7 & 519.5 & 40.8 & 476.2 & 2.5 \\
\hline & 505.5 & 553.1 & 47.6 & 512.9 & 7.4 \\
\hline & 874.3 & 850.0 & 24.3 & 879.5 & 5.2 \\
\hline Average & & & 38.0 & & 19.6 \\
\hline
\end{tabular}

*: from Ref. 3 


\section{S2 GENERATION OF OLIVINIC NPS}

\section{S2.1 Bulk cut NPs}

Bulk cut (BC) olivine (Ol) NPs (NPs) have been generated following the top-down approach briefly described in Subsection 3.1.1 Here we provide a more detailed explanation. The steps are:

- we previously optimized at PBE/OIBS level the crystallographic structure of pure forsterite unit cell (Figure 2, panel A, top-left corner); ${ }^{2}$

- the optimized unit cell structure has been then replicated several times along the three periodic directions;

- exploiting some features of the VESTA software, ${ }^{4}$ we cut along the crystallographic directions representing the most stable surfaces in forsterite natural crystals. ${ }^{5}$ In order to cut, the Miller's index $(h k l)$ and the distance from the centre of the NP of each selected plane are required, with the latter being proportional to the surface energy values $E_{S} .{ }^{6}$ The $(h k l)$ indices and the corresponding $E_{S}$ values we used are listed in Table S4: they derived from previous calculations at an higher level of theory than the present work, where we energetically and structurally characterized the main forsterite surfaces, ${ }^{7}$ and are in good accordance with other computational results present in literature. ${ }^{8,9}$

Using distances proportional to the $E_{S}$ values in Table S4, we cut forsterite bulk adopting the following criteria:

- all NPs must be electrically neutral (i.e., with a Mg:Si:O stoichiometry of 2:1:4) for a proper comparison with the forsterite bulk energetics;

- all the silicate units must be kept intact, i.e., covalent $\mathrm{Si}-\mathrm{O}$ bonds must be preserved;

- starting from the smaller NP of 98 atoms $(N=14$ formula-units), we generated larger NPs of increasing size, up to 812 atoms ( $N=116$ formula-units), with steps of about 100 atoms. We also generated a huge NP of 2926 atoms $(N=418$ formula unit). See Table 1 of the article for the full list of all NPs.

Despite the first criterion, the very initial cuts are not, in general, electrically neutral because of two reasons. Firstly, the forsterite unit cell presents an inversion centre on a $\mathrm{Mg}$ ion, thus all cut NPs still have this inversion centre and the number of $\mathrm{Mg}$ ions is odd. This can be easily fixed by eliminating one $\mathrm{Mg}$ ion (so that removing any residual symmetry of the Ol NPs), or by translating the unit cell of half the length of one lattice vector (so that the inversion centre is not placed on an atom anymore). Nevertheless, even after this removal/translation, NPs can still be electrically charged (with charge values up to $\pm 20-30$ atomic units), thus further modifications are also required, such as removal of some $\mathrm{SiO}_{4}$ units and $\mathrm{Mg}$ ions and/or alterations of the perfect crystalline ratios among the $E_{S}$ values of Table S4. Ol NPs generated after the unit cell translation have been labelled with "trasl". The Ol NPs generated with the top-down approach and these a posteriori modification represent our initial set (Figure S1) and closely resemble the Wulff shape of forsterite crystals 
(compare the Wullf shape in the top-right corner of panel A of Figure 2, with the structures reported Figure S1).

However, the initial NPs of Figure S1 have not been directly optimized at the PBE/OlBS level, because of problems encountered in the convergence of the electronic energy. We rationalized this behaviour by the fact that in the very initial crystalline cuts, several exposed $\mathrm{Mg}$ ions are under-coordinated, with respect to the coordination number of $\mathrm{Mg}$ in forsterite bulk (i.e. 6). Attempts to use semi-empirical electronic structure methods to optimise these NP with the MOPAC $\operatorname{code}^{10}$ were not successful. Therefore, we decided to employ classical force-field (FF) calculations. For these calculations, we adopted the GULP code, ${ }^{11}$ exploiting several different available FFs. The idea of these "pre-optimizations" was to partially relax the atomic positions, thus furnishing better structures for the subsequent QM optimizations. Among all tested FFs, only Reax FF gave acceptable results, ${ }^{12}$ i.e., we did not observe any breaking of Si-O bonds and/or any ejection of atoms from the NP. Thus, the Reax FF pre-optimized structures were submitted to PBE/OlBs optimization with the CRYSTAL code, with the exception of the 418 unit NP that has been submitted to Walker FF optimization only. The PBE/OlBS-optimized NP structures (Walker FF-optimized for the 418 BC NP) are reported in Figure S2.

Table S4 Miller's indices $(h k l)$ and computed surface energies $E_{S}$ of the main surfaces of forsterite (from Ref. 7). \% A is the extension percentage of a given surface with respect to the total area of a macroscopic perfect crystal computed according to the Gibbs-Wulff theorem. ${ }^{6}$

\begin{tabular}{lrr}
\hline$(h k l)$ & $E_{S}\left(\mathrm{~J} \mathrm{~m}^{-2}\right)$ & $\% \mathrm{~A}$ \\
\hline$(010)$ & 1.44 & 23.8 \\
$(120)$ & 1.68 & 29.7 \\
$(101)$ & 1.96 & 20.7 \\
$(001)$ & 1.98 & 19.8 \\
$(111)$ & 2.19 & 2.5 \\
$(021)$ & 2.24 & 3.5 \\
$(110)$ & 2.47 & 0.0 \\
\hline
\end{tabular}



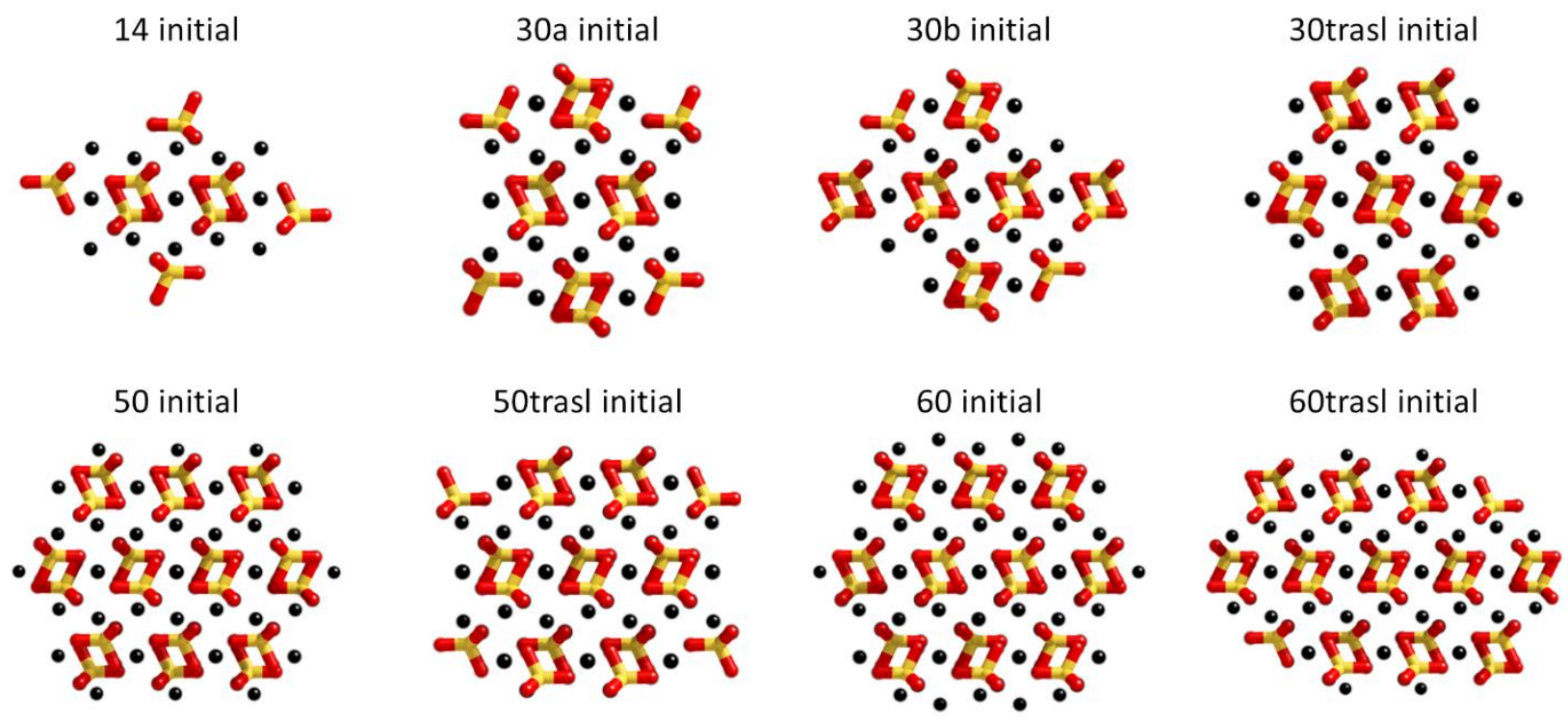

80 initial

80trasl initial

92 initial

92trasl initial

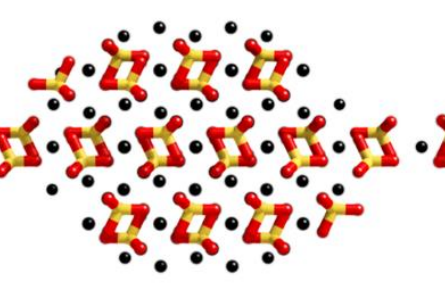

Fo.

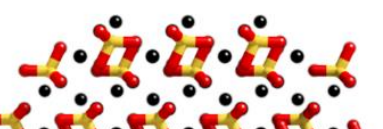

rofic

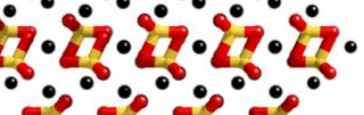

H.
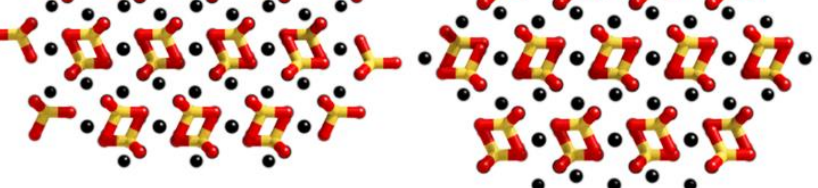

104 initial

104trasl initial

116 initial

r. 0.0 .9
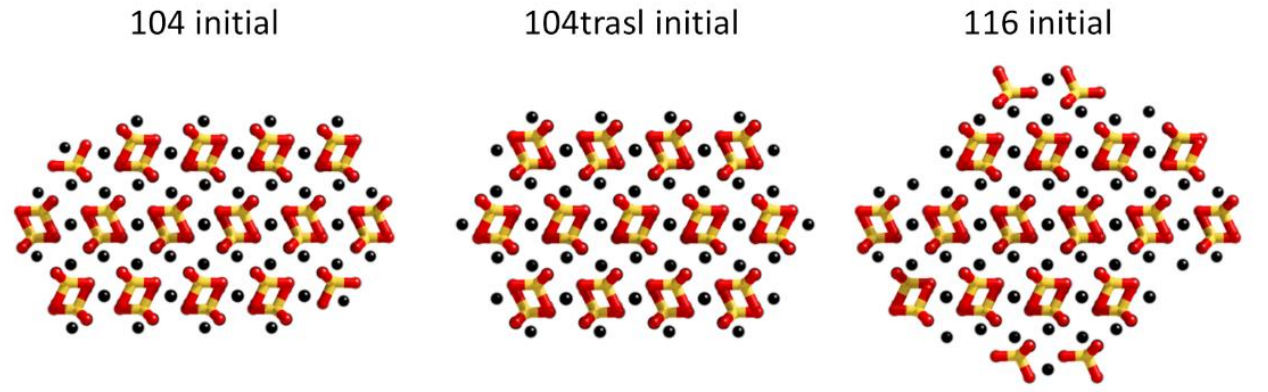

116 trasl initial

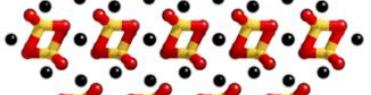

- $0^{\circ} 0^{\circ}$

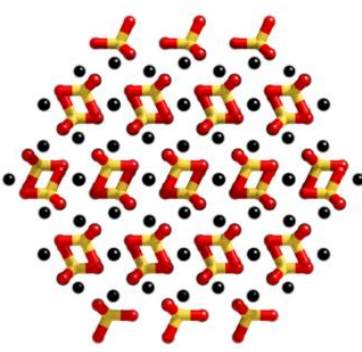

418 initial

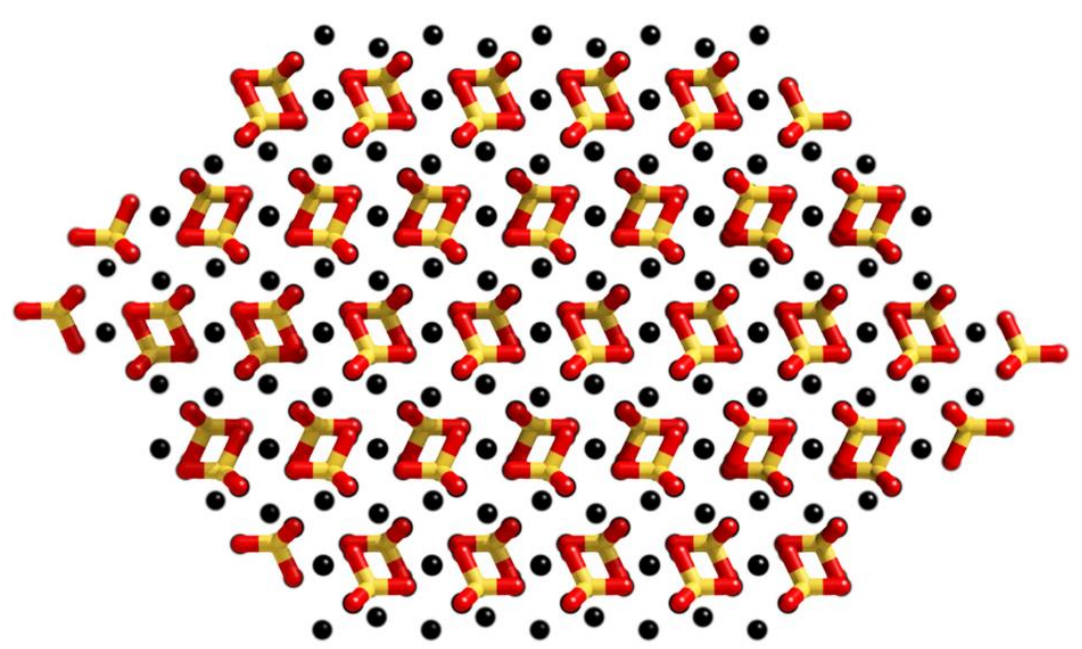

Figure S1 Graphical representation of all the initial structures of our Ol NPs. Colour scheme: oxygen in red, silicon in yellow, magnesium ions in black. Sizes are not to scale. 


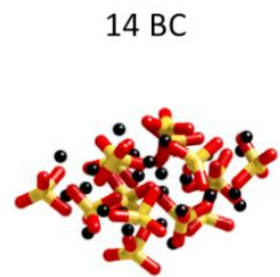

$50 \mathrm{BC}$

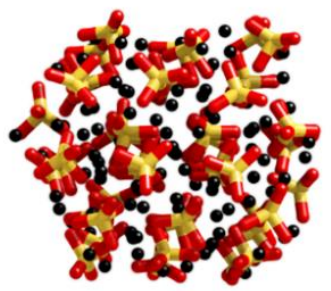

$80 \mathrm{BC}$

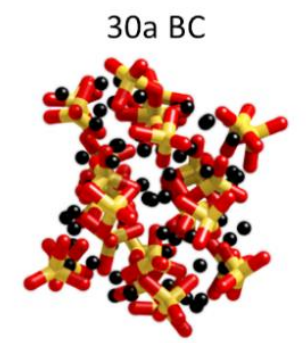

50trasl BC

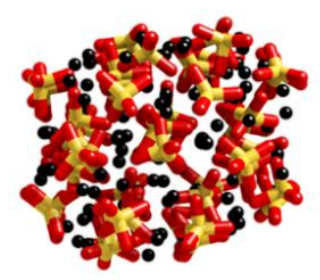

80trasl BC

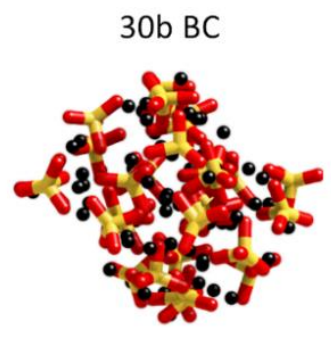

60 initial

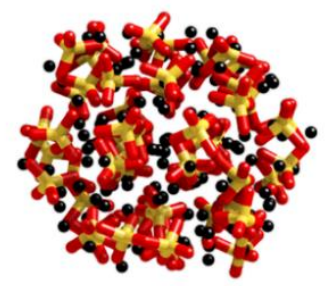

92 initial

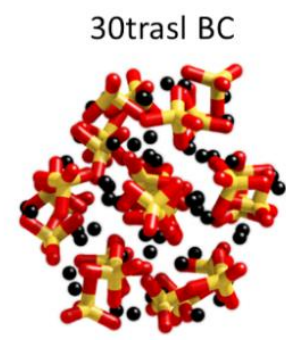

60trasl BC

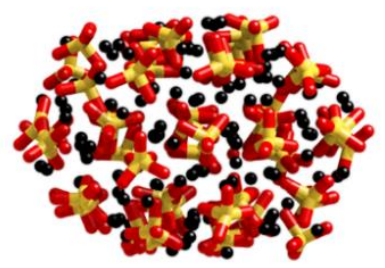

92trasl BC

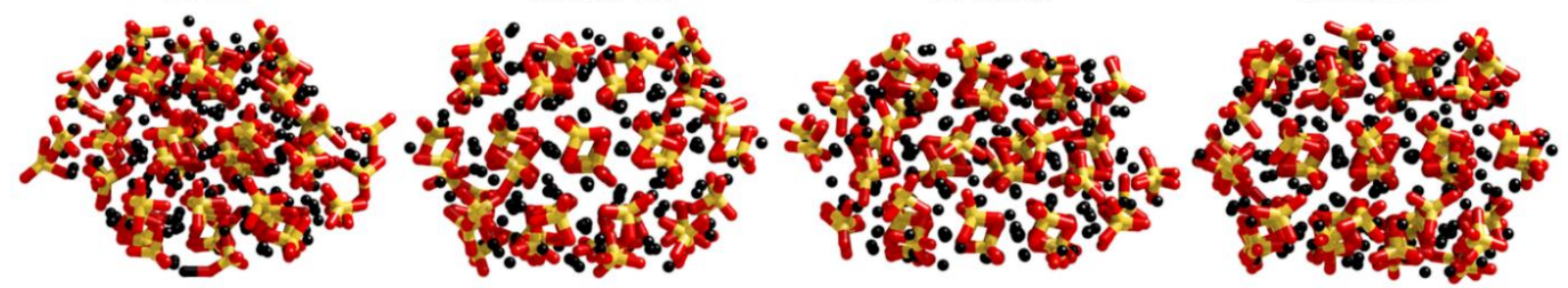

104 BC

104trasl BC

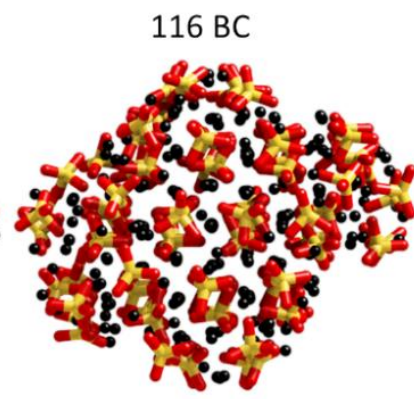

116 BC

116trasl BC

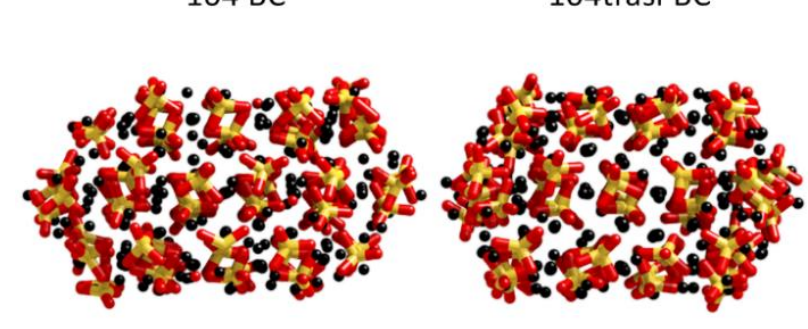

$418 \mathrm{BC}$
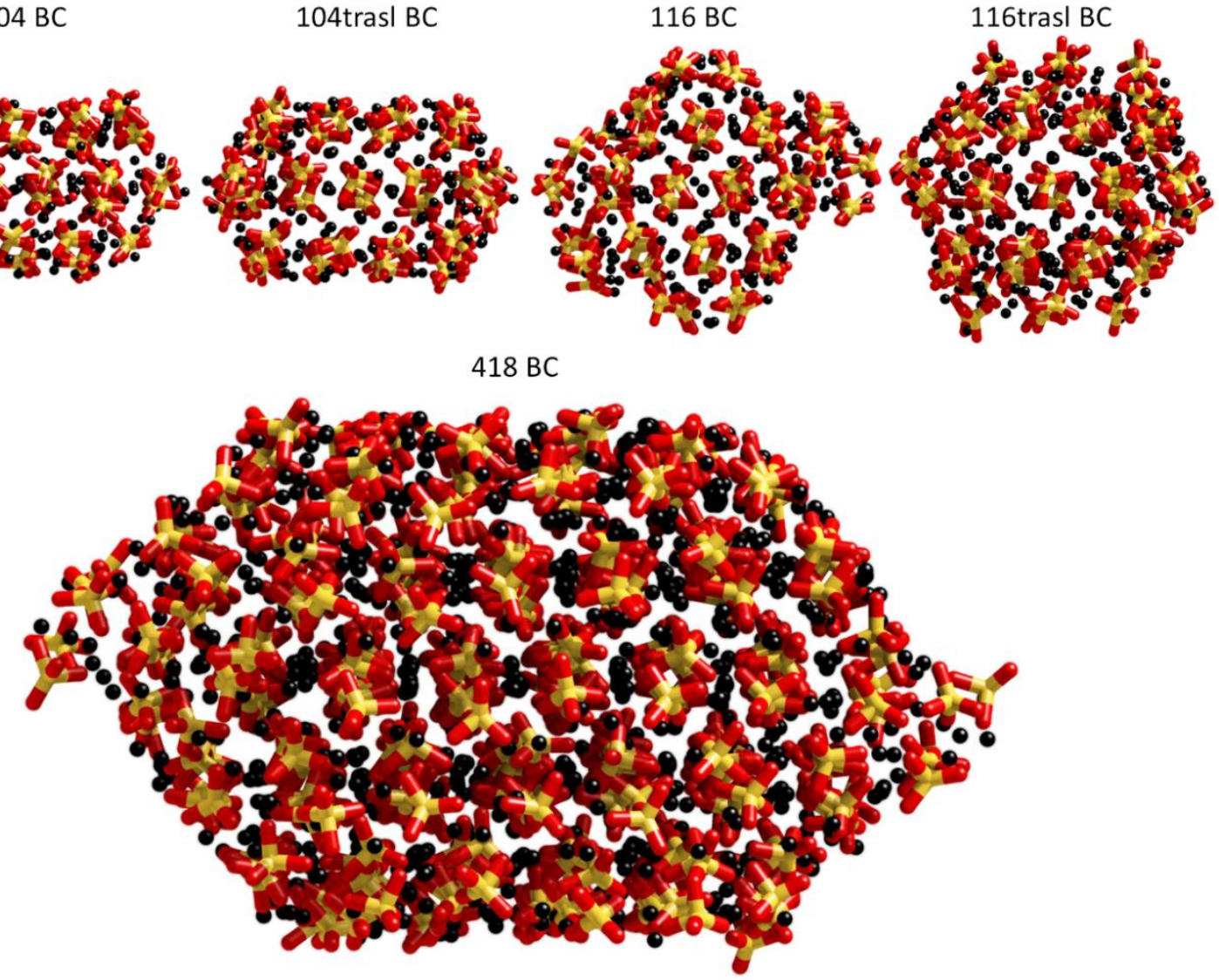

Figure S2 Graphical representation of all the bulk cut (BC) Ol NPs after PBE/OlBS optimizations. Colour scheme: oxygen in red, silicon in yellow, magnesium ions in black. Sizes are not to scale. 


\section{S2.2 Amorphized NPs}

In this Subsection, we justify the choice of 20 ps as production time for all the Ol NPs amorphizations runs, regardless the size (for $14 \leq N \leq 16$ ).

To estimate the degree of amorphization of amorphized NPs after $20 \mathrm{ps}$ as MD production time, we used the root-mean-square-displacements of the silicon atoms (RMSD-Si) with respect to the very initial cuts (i.e., the initial geometries in Figure S1). Specifically, we computed RMSD-Si values after the fixed time of 20 ps we selected and after increasing production time with size. Data for some NPs are resumed in Table S5 as illustrative examples. Increasing the production time with size does not, in general, produce very larger RMSD-Si values. Indeed, in some cases (92trasl A and 104 trasl), these values do not significantly change, while for the 80trasl A NP it is slightly lower even if the production time increased by a factor 5 (from 20 to $100 \mathrm{ps}$ ). This can be rationalized by the fact that, in terms of dimensions, our Ol NPs do not spread in a large range (from about 0.6 to about $1.4 \mathrm{~nm}$ in radius). The PBE/OlBS-optimized (Walker FF-optimized for the 418 A NPs) A Ol NP structures are reported in Figure S3.

Table S5 Root-mean-square-displacements (in $\AA$ ) of silicon atoms (RMSD-Si) after Walker FF molecular dynamics simulations, for some selected NPs. Second column: RMSDs-Si after a fixed time of 20 ps. Third column: RMSDs-Si after increasing production time (in round brackets) with size.

\begin{tabular}{lrr}
\hline NP & RMSD-Si after 20 ps MD & RMSD-Si after $x$ ps MD \\
\hline 50trasl A & 2.04 & $3.04(60 \mathrm{ps})$ \\
60trasl A & 1.70 & $2.33(80 \mathrm{ps})$ \\
80trasl A & 2.26 & $2.11(100 \mathrm{ps})$ \\
92trasl A & 2.15 & $2.59(120 \mathrm{ps})$ \\
104trasl A & 2.18 & $2.60(150 \mathrm{ps})$ \\
\hline
\end{tabular}


$14 \mathrm{~A}$

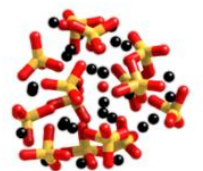

$50 \mathrm{~A}$

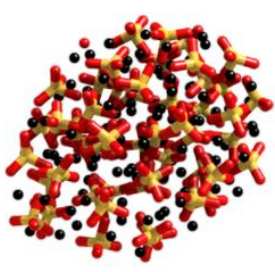

$80 \mathrm{~A}$

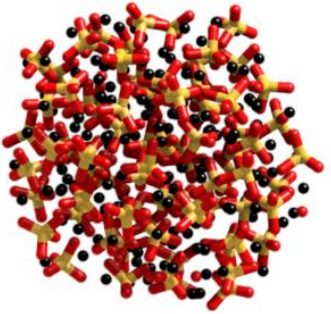

$104 \mathrm{~A}$

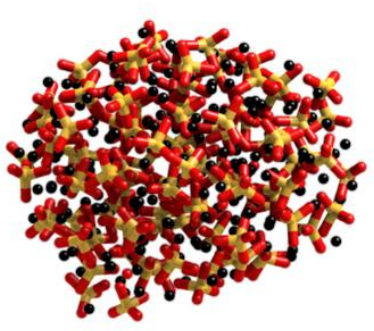

$30 a \mathrm{~A}$ at $1800 \mathrm{~K}$

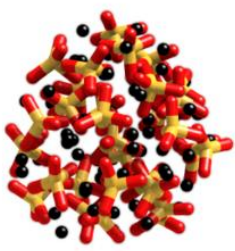

50trasl A

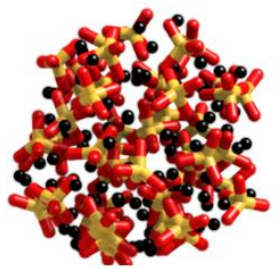

80trasl A

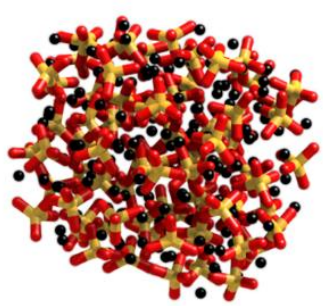

104trasl A

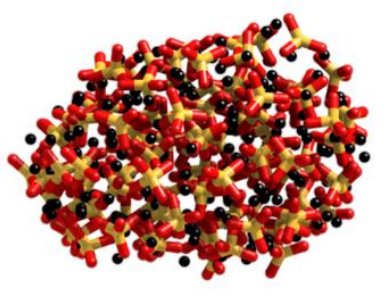

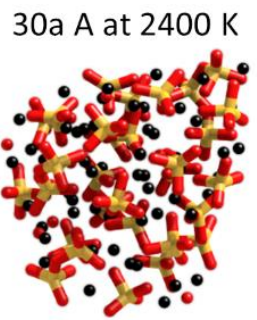

$60 \mathrm{~A}$

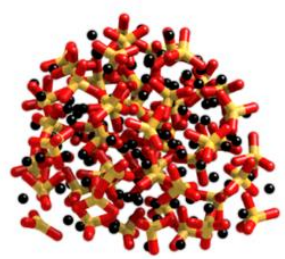

$92 \mathrm{~A}$

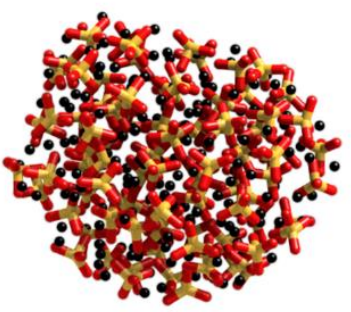

$116 \mathrm{~A}$

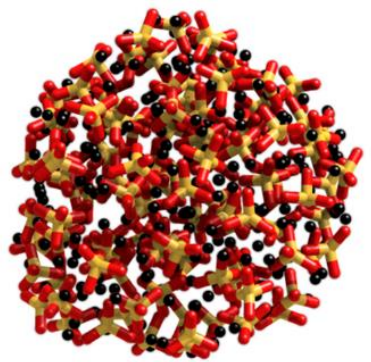

$18 \mathrm{~A}$

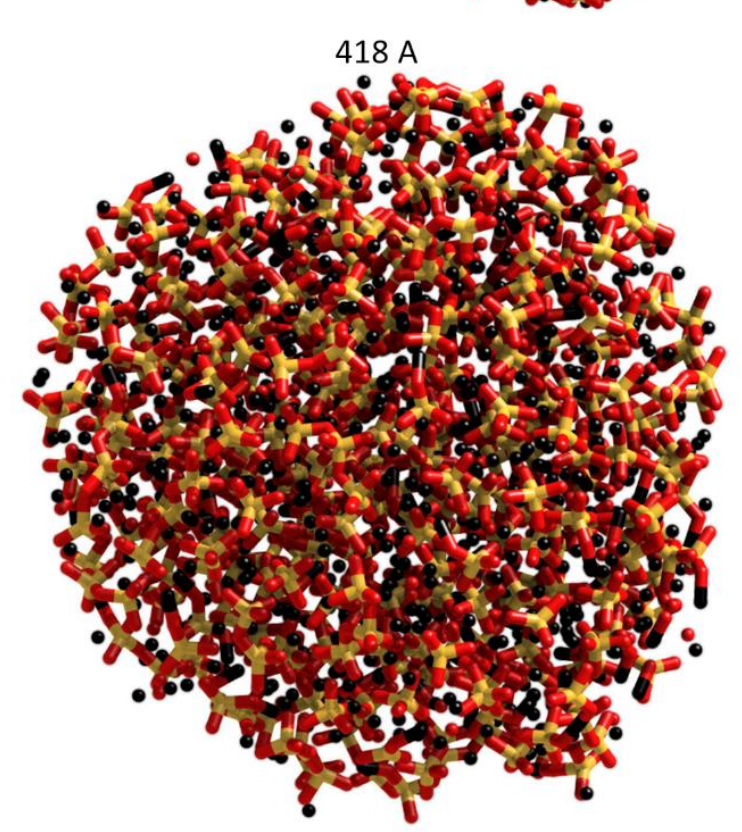

30trasl A

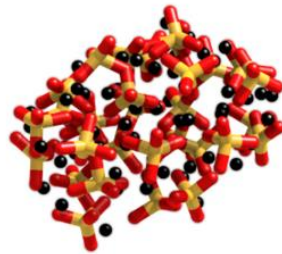

60trasl A

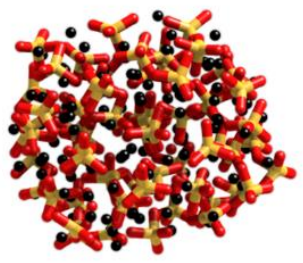

92trasl A

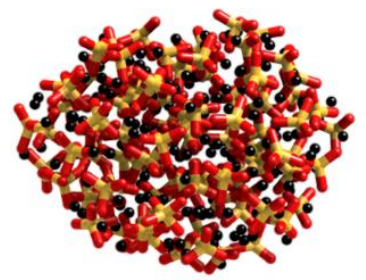

116trasl A

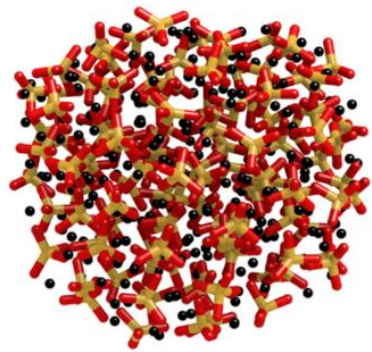

Figure S3 Graphical representation of all the amorphized (A) Ol NPs after PBE/OIBS optimizations. Colour scheme: oxygen in red, silicon in yellow, magnesium ions in black. Sizes are not to scale. 


\section{S2.3 Nucleated NPs}

Taking advantage of the ionic nature of the compounds, the small size of the NPs involved in this work and previous theoretical models on the nucleation of stardust silicates, ${ }^{13}$ we generated NPs by aggregation of magnesium, oxygen and silicon monoxide $(\mathrm{SiO})$ - our "monomeric" species - into a seed NP using MD simulations with the LAMMPS code. ${ }^{14}$ Overall, the process involves the individual addition of monomeric species using random orientations and impact parameters, with an incoming velocity extracted from a Boltzmann distribution for a temperature of $1800 \mathrm{~K}$, with a short time to allow atomic reorganization. Each monomeric addition consists of an MD run of 4.5 ps including equilibration, monomeric addition and relaxation. The PBE/OlBS-optimized N Ol NP structures are reported in Figure S4.

$14 \mathrm{~N}$

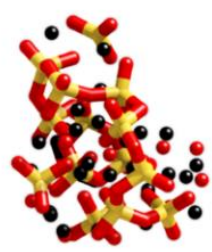

$60 \mathrm{~N}$

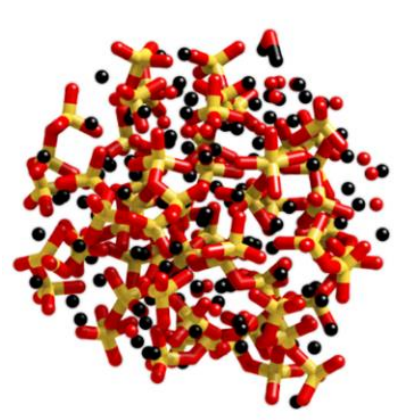

$30 \mathrm{~N}$

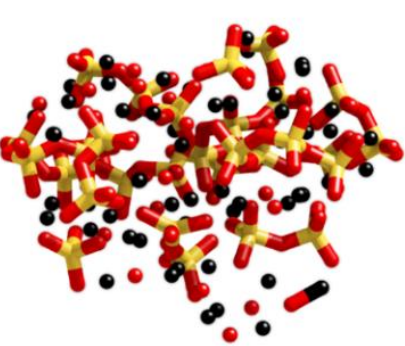

$80 \mathrm{~N}$

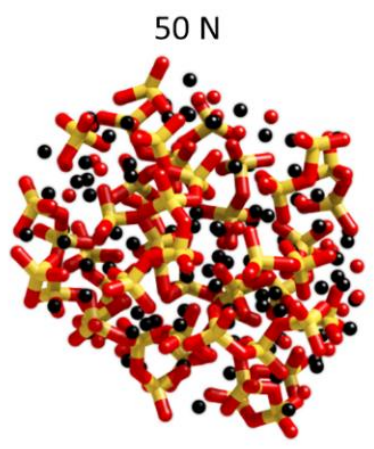

$92 \mathrm{~N}$

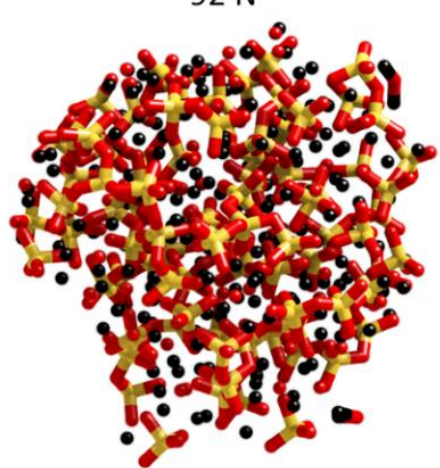

$104 \mathrm{~N}$
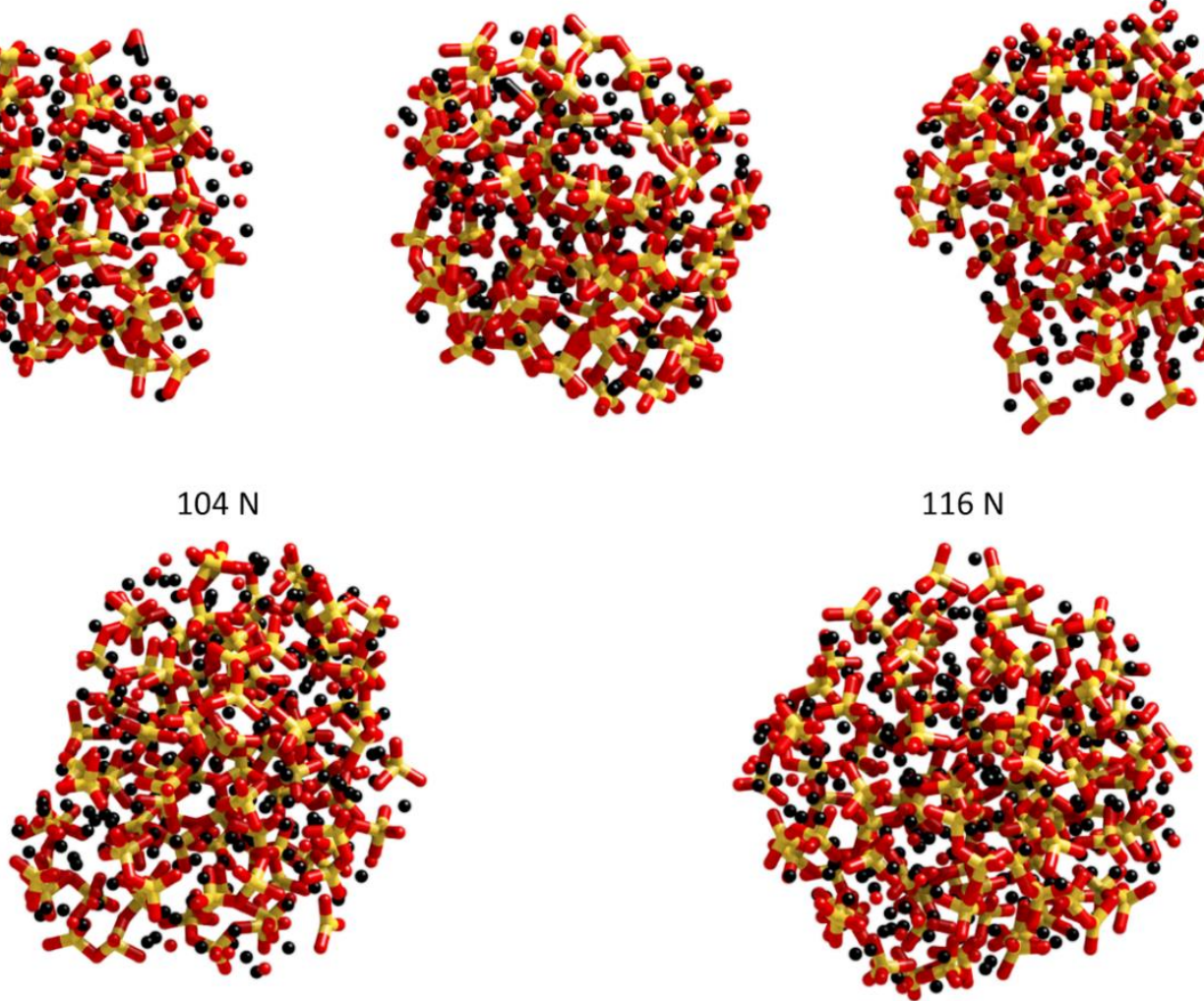

Figure S4 Graphical representation of all the nucleated (N) Ol NPs after PBE/OlBS optimizations. Colour scheme: oxygen in red, silicon in yellow, magnesium ions in black. Sizes are not to scale. 
In this Section, we provide a short theoretical explanation of the procedure we followed for obtaining the IR spectra from molecular dynamics (MD) simulations. For more details, please refer to Ref. 15.

To obtain IR spectra from MD simulations, one needs to compute the electric dipole moment of the system at each step $t$ of the MD simulation. From the time evolution of such dipole moment, the dipole autocorrelation function $C_{\mu}(t)$ can be defined as

$$
C_{\mu}(t)=<\vec{\mu}(0) \cdot \vec{\mu}(t)>
$$

i.e., as the average between $\vec{\mu}(0)$ and $\vec{\mu}(t)$. The dipole moments are directly computed from the electric charges $\left(q_{i}\right.$, centred at positions $\left.\vec{r}_{i}(t)\right)$ distribution at a given time $t$, i.e.

$$
\vec{\mu}(t)=\sum_{i} q_{i} \vec{r}_{i}(t)
$$

and analogously for $\vec{\mu}(0)$.

Starting from the vibrational transition rules, it is possible to prove that the absorption cross section $\alpha(\omega)$ can be obtained as follows ( $\omega$ is the angular frequency)

$$
\alpha(\omega)=\frac{4 \pi^{2}\left(1-e^{-\beta \hbar \omega}\right)}{3 \hbar c n} I(\omega)
$$

where $I(\omega)$ is the lineshape, $\beta=k_{B} /_{T}$ ( $k_{B}$ is the Boltzmann's constant), $T$ is the temperature, $\hbar$ is the reduced Planck's constant, $c$ is the speed of light, and $n$ is the index of refraction of the medium (considered to be equal to 1$)$.

To obtain $I(\omega)$, the Fourier Transform of the dipole auto-correlation function must be computed, i.e.

$$
I(\omega)=\frac{1}{2 \pi} \int_{-\infty}^{\infty} d t e^{-i \omega t} C_{\mu}(t)
$$

In order to obtain cleaner results, we also applied a filter to the obtained IR spectra to smooth it. For the O1 NPs case, we applied a Savitzky-Golay filter.

These MD simulations have been conducted with the GULP code, at Walker FF level. The simulations consisted in an equilibration phase of $5 \mathrm{ps}$ followed by a production of $100 \mathrm{ps}$ with a timestep of $0.5 \mathrm{fs}$ in both stages. To compute the dipole autocorrelation function, we averaged the multiplication of the dipole moments for every step of the dynamics using a shift in time.

For example:

The first step consists in computing the average of the $\vec{\mu}(t) \cdot \vec{\mu}(t)$ product, i.e., we multiplied the electric dipole moment by itself and then take the average.

In the second step, we compute the $\vec{\mu}(t) \cdot \vec{\mu}(t+\Delta t)$ product ( $\Delta t=.5 \mathrm{fs})$ then take the average. The same procedure is followed for $2 \Delta t$, and for each subsequent time shift. 
In this Section, we provide the full representation of all IR harmonic spectra obtained from both DFT calculations using CRYSTAL and FF calculations using GULP, as well as a comparison between selected CRYSTAL vs GULP IR spectra. CRYSTAL intensities are in $\mathrm{km} \mathrm{mol}^{-1}$, while GULP intensities are in atomic units squared. Where spectra are compared, we normalised the height of highest peak in both spectra to 1 and intensity units are arbitrary. For all spectra, the full width at half maximum broadening for individual peaks is set equal to $10 \mathrm{~cm}^{-1}$, as justified in the main text.

\section{S5.1 CRYSTAL spectra}

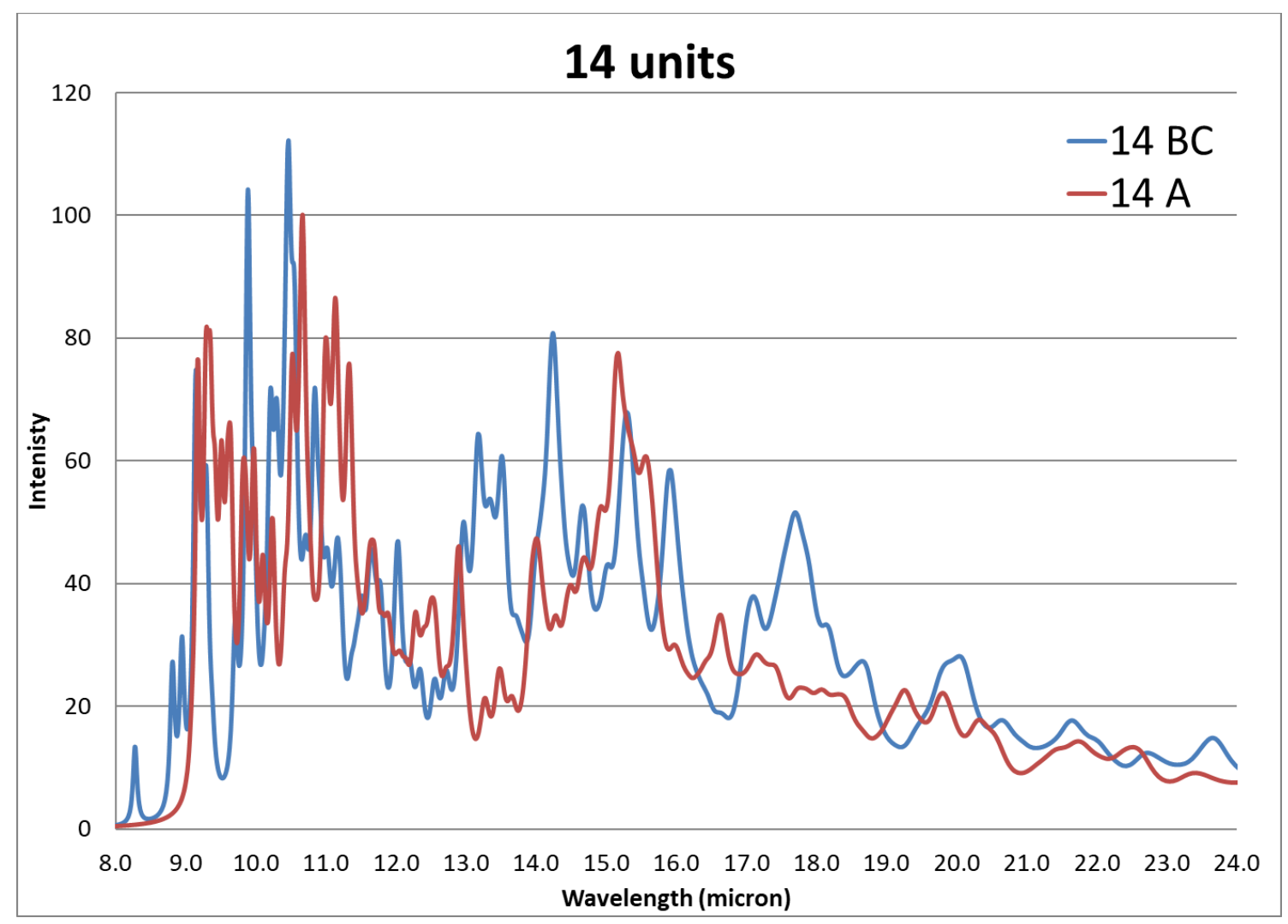

Figure S5 CRYSTAL simulated IR spectra for all the 14 formula unit NPs. 


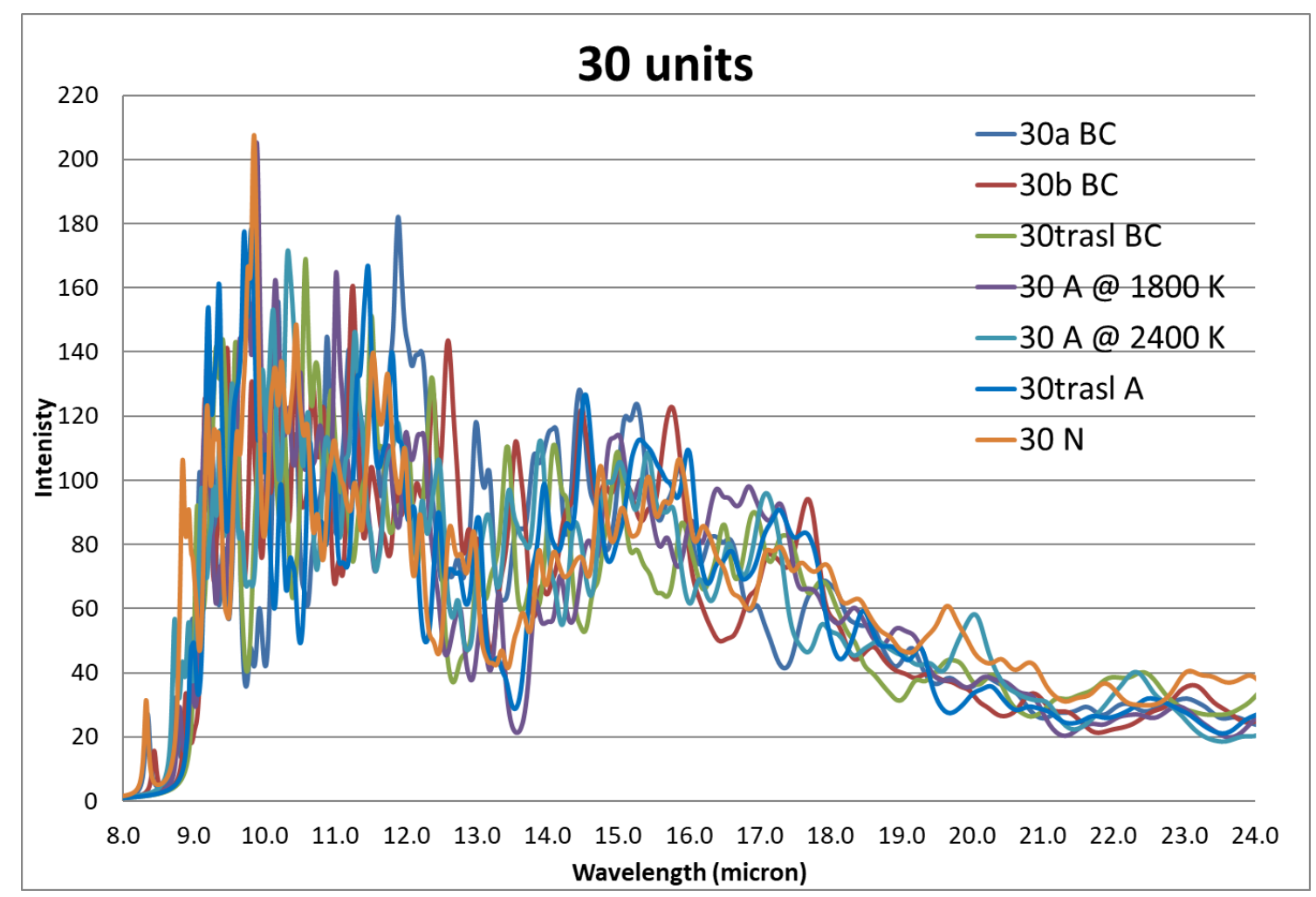

Figure S6 CRYSTAL simulated IR spectra for all the 30 formula unit NPs.

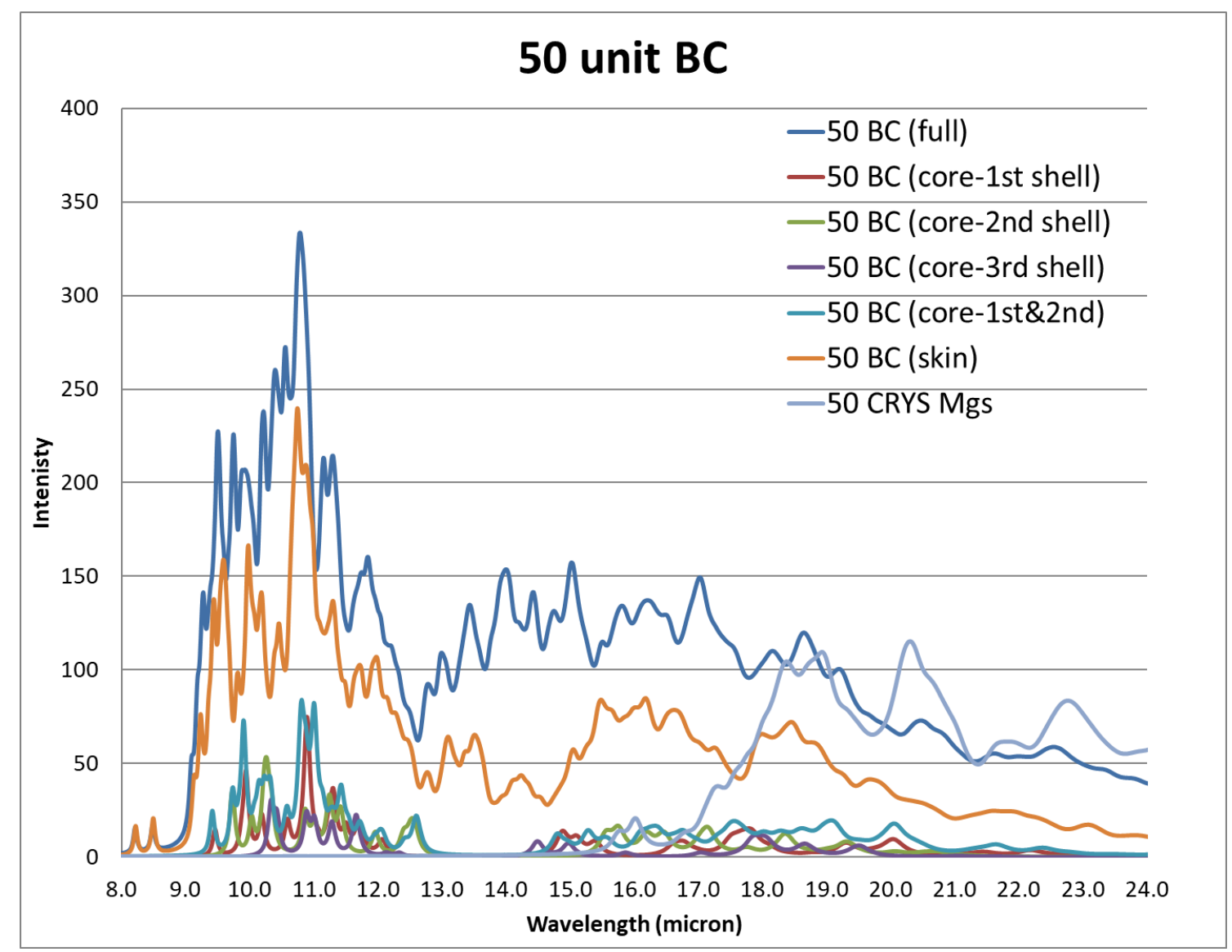

Figure S7 CRYSTAL simulated IR spectra for all the 50 BC NP: full spectrum and contributions from subsets of atoms in the NP. 
S5.2 CRYSTAL-GULP comparison

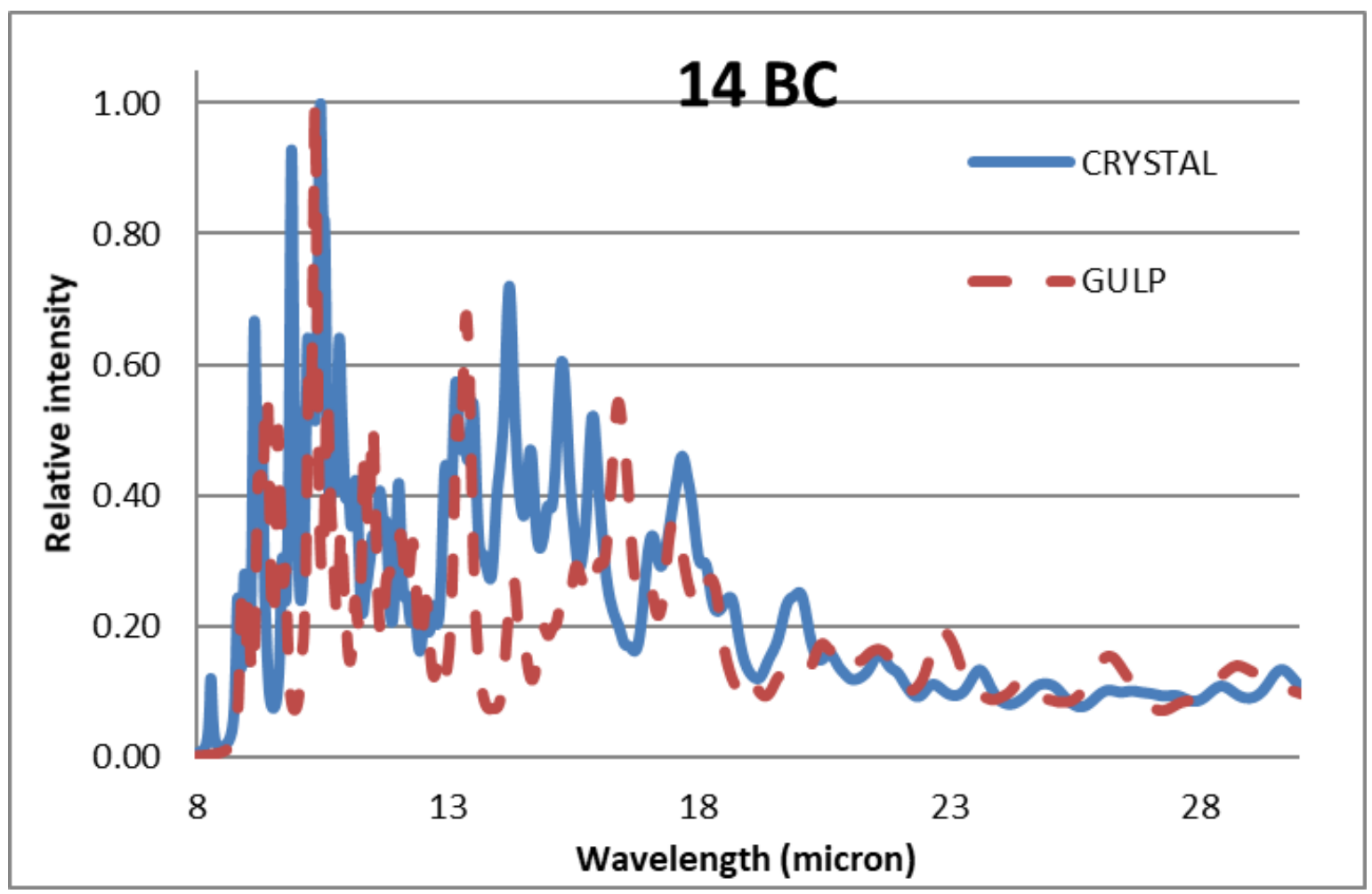

Figure S8 CRYSTAL vs GULP: IR spectra comparison for the 14 BC NP.

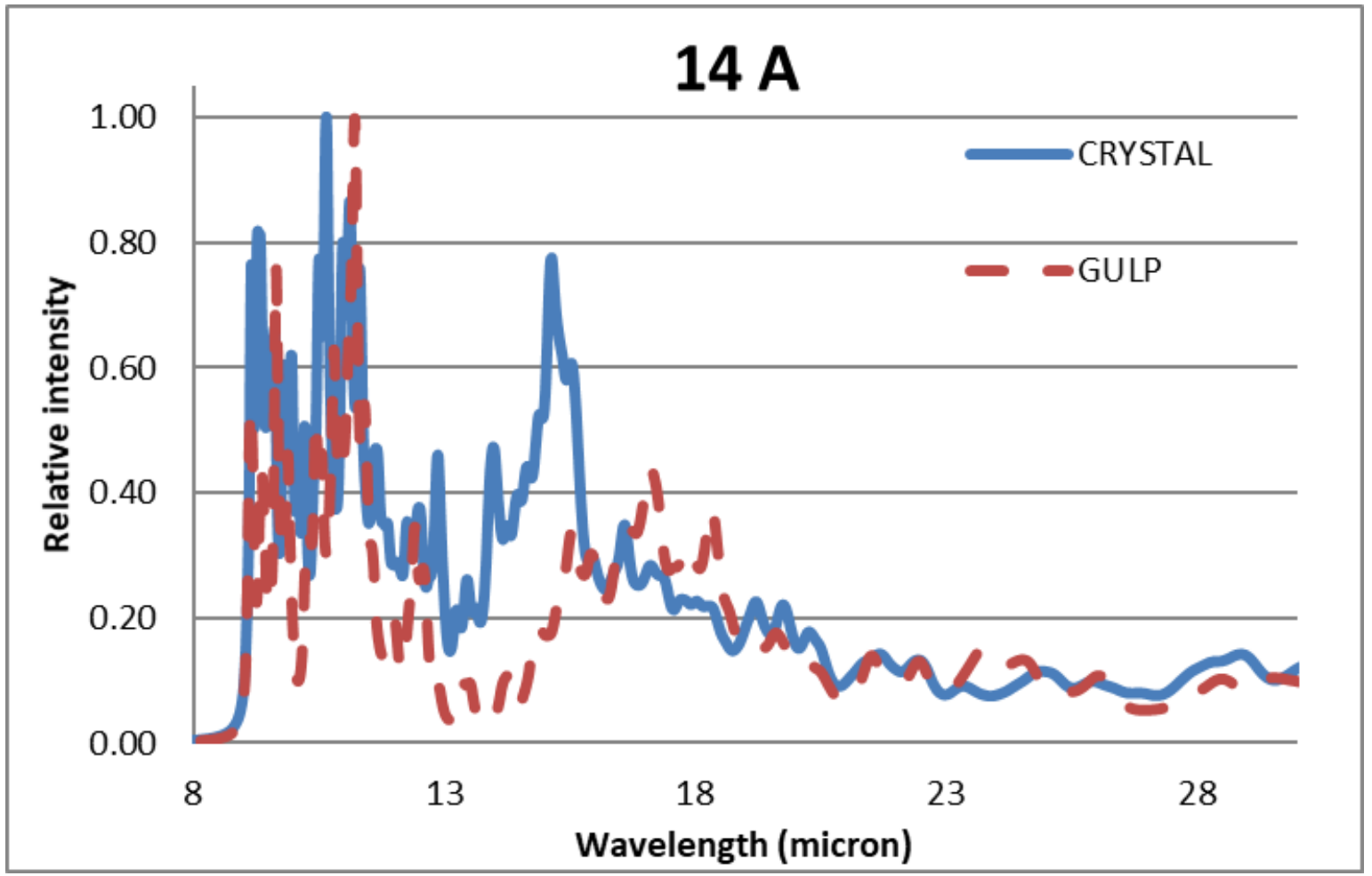

Figure S9 CRYSTAL vs GULP: IR spectra comparison for the 14 A NP. 


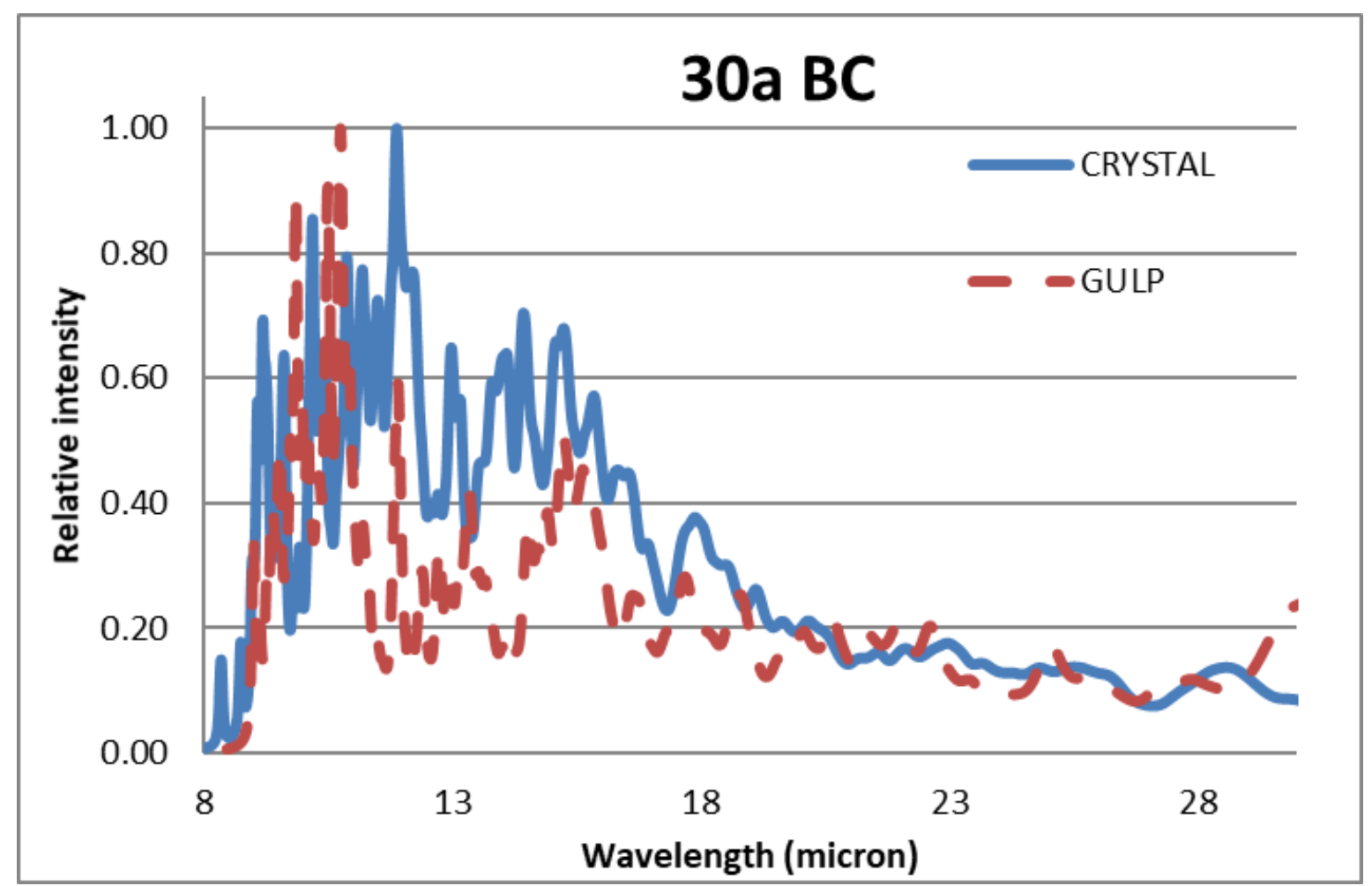

Figure S10 CRYSTAL vs GULP: IR spectra comparison for the 30a BC NP.

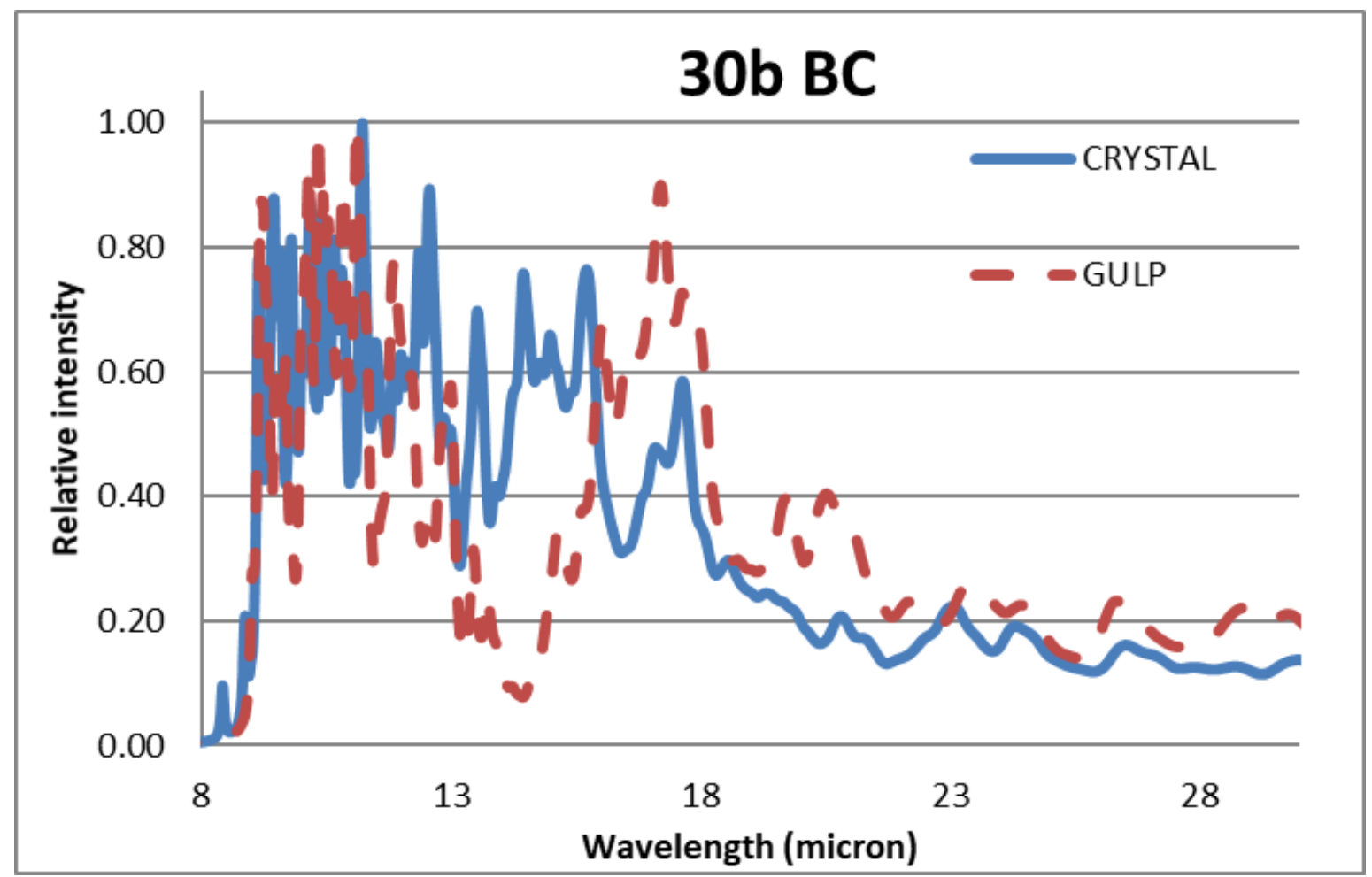

Figure S11 CRYSTAL vs GULP: IR spectra comparison for the 30b BC NP. 


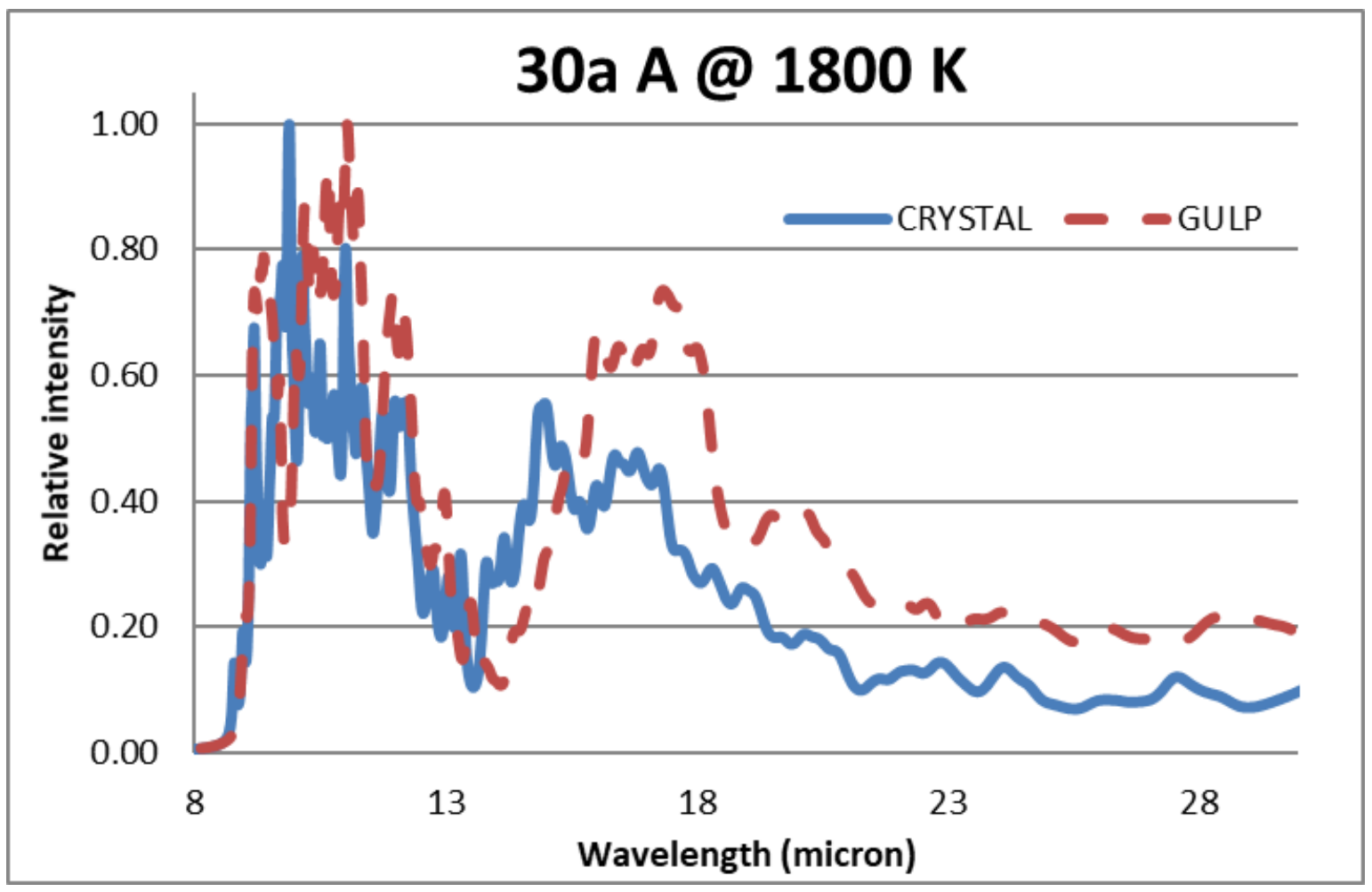

Figure S12 CRYSTAL vs GULP: IR spectra comparison for the 30a A at $1800 \mathrm{~K}$ NP.

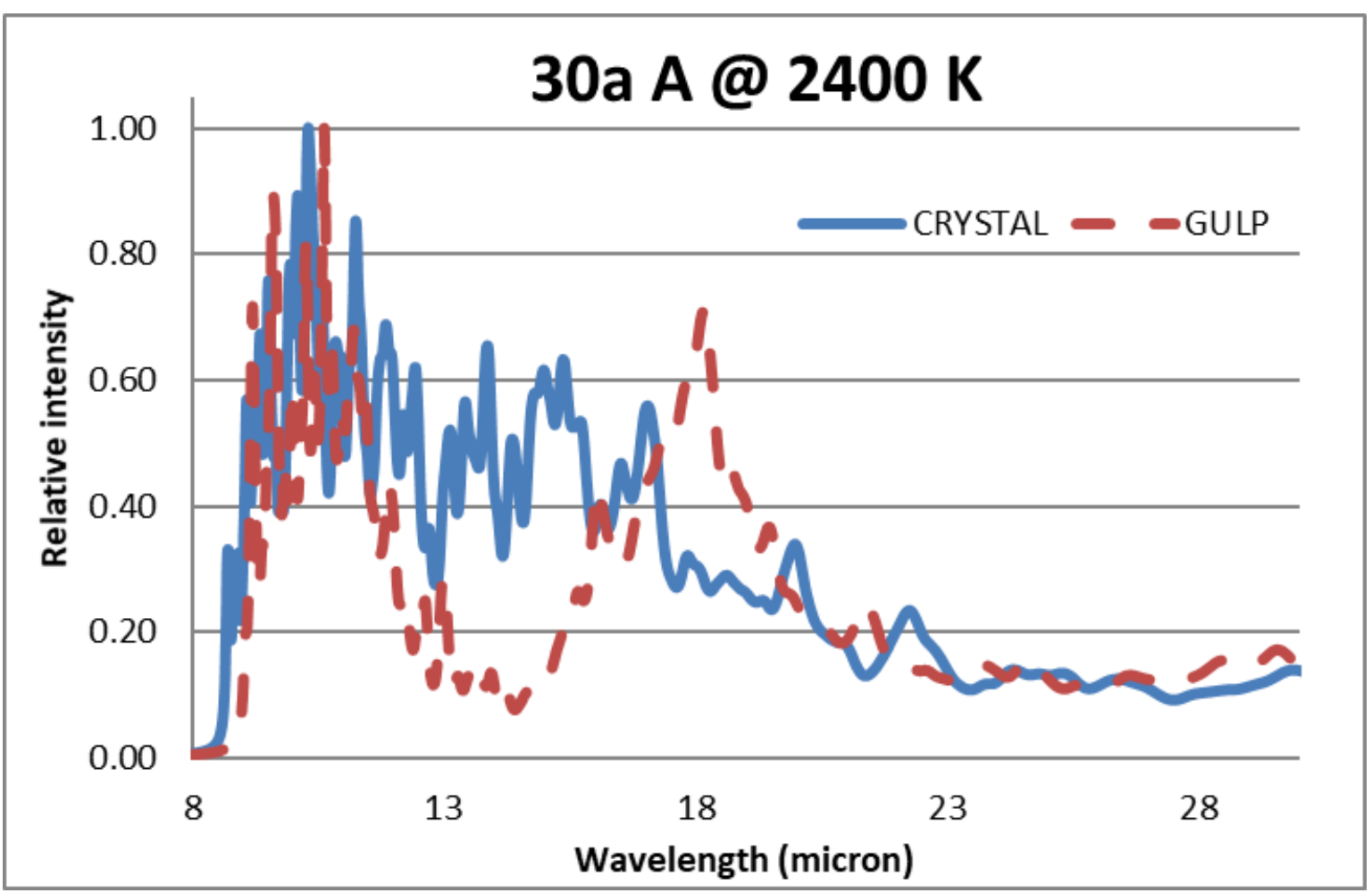

Figure S13 CRYSTAL vs GULP: IR spectra comparison for the 30a A at $2400 \mathrm{~K}$ NP. 


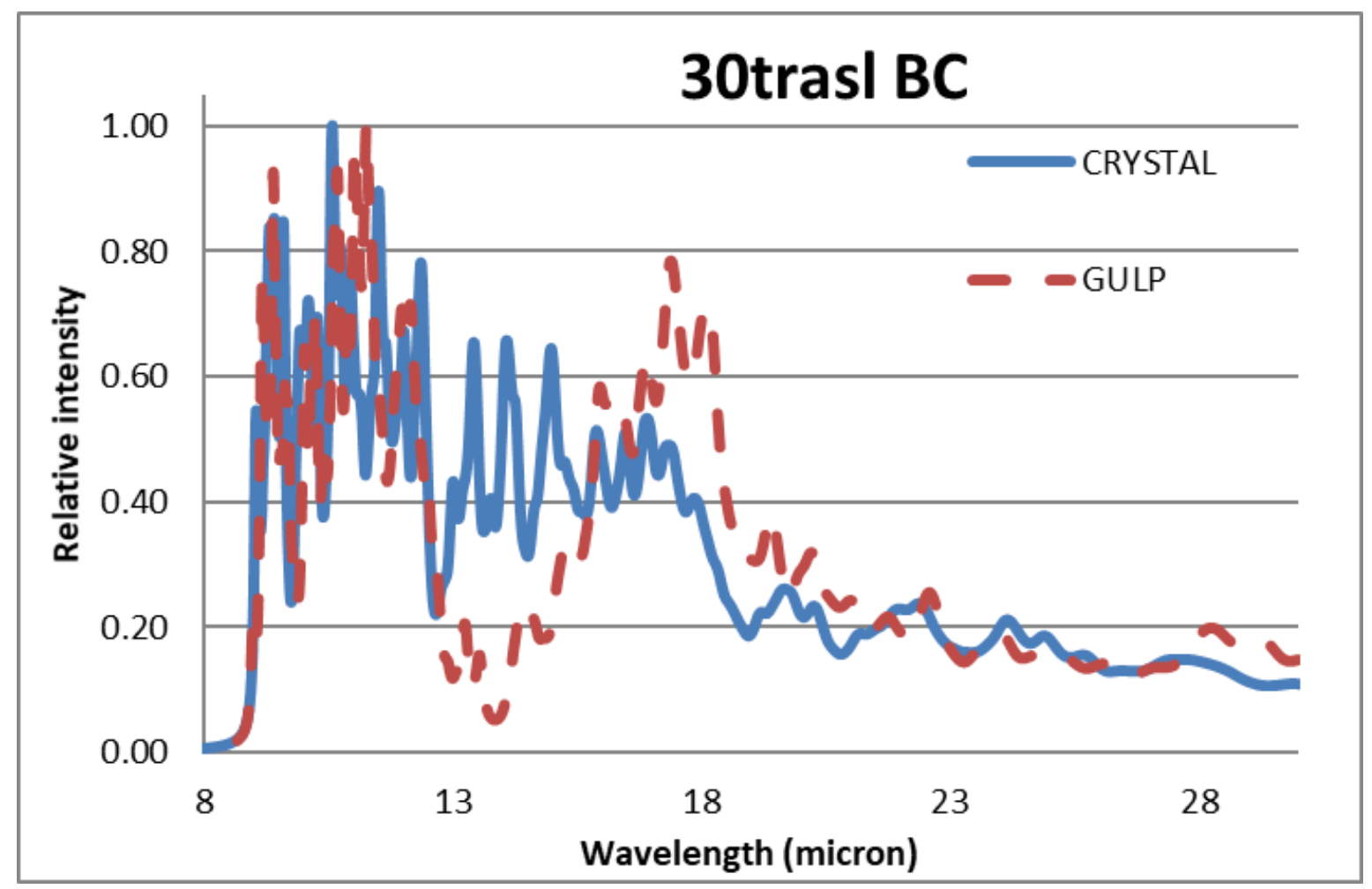

Figure S14 CRYSTAL vs GULP: IR spectra comparison for the 30trasl BC NP.

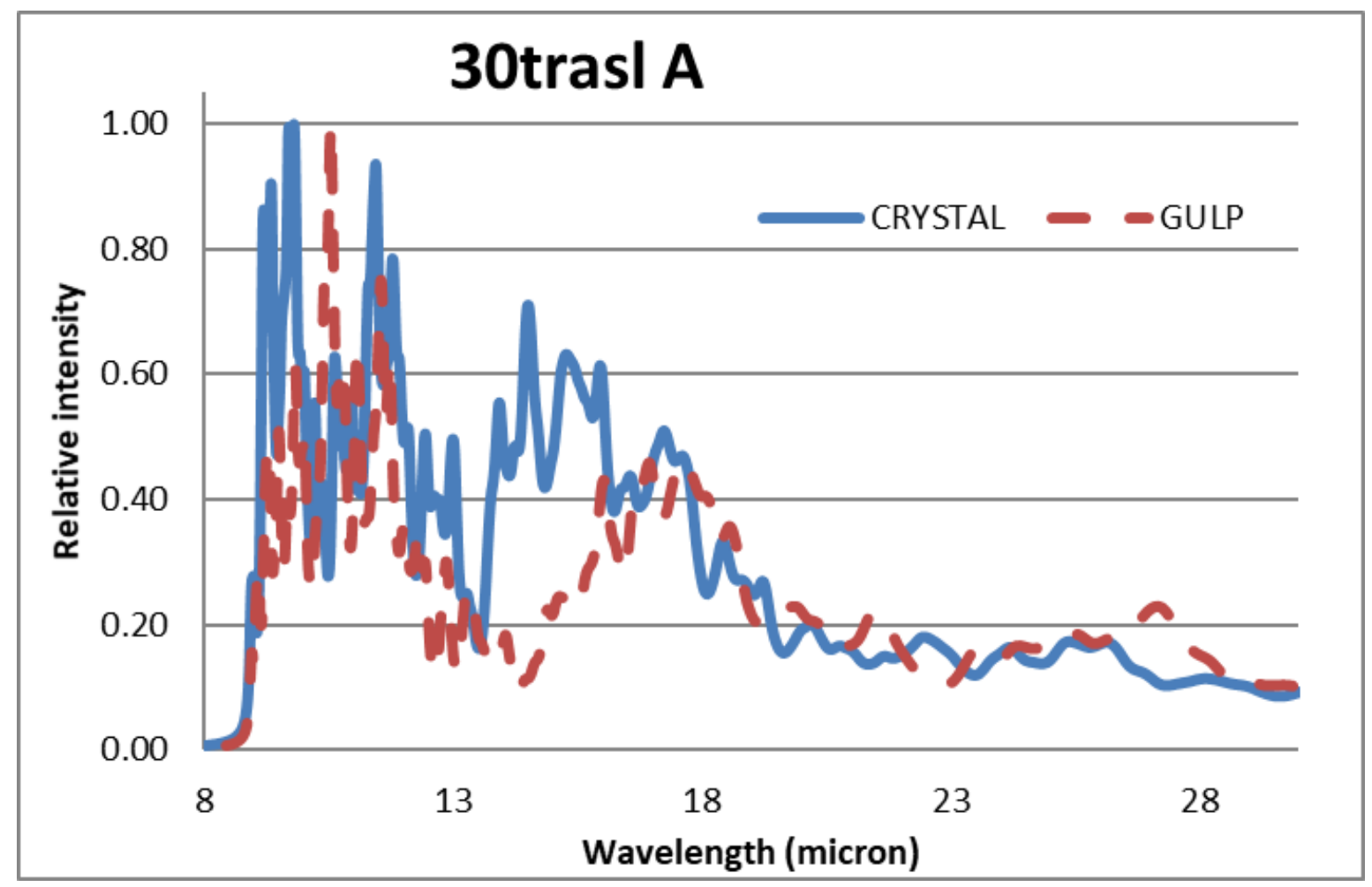

Figure S15 CRYSTAL vs GULP: IR spectra comparison for the 30trasl A NP. 


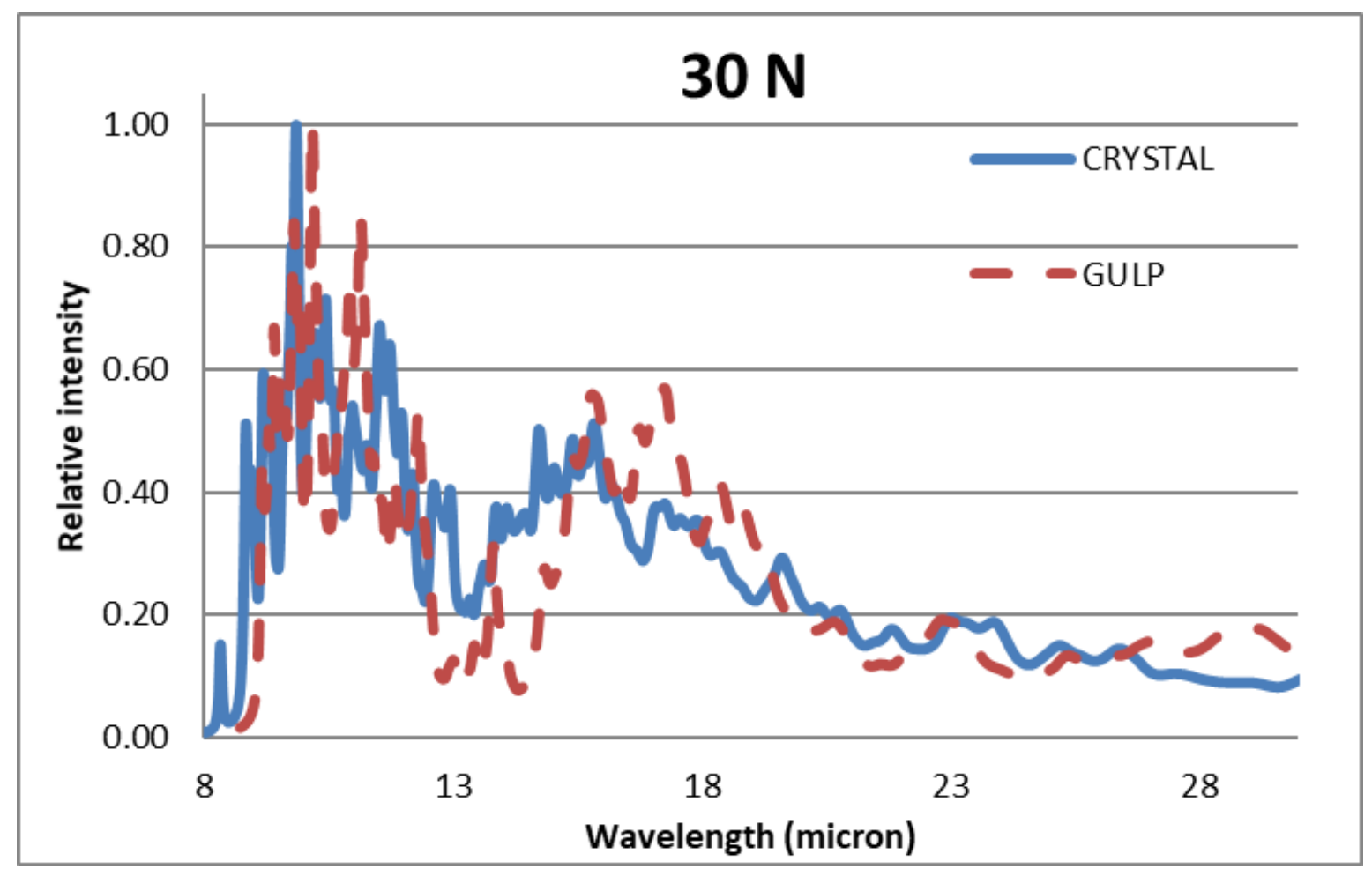

Figure S16 CRYSTAL vs GULP: IR spectra comparison for the 30 N NP.

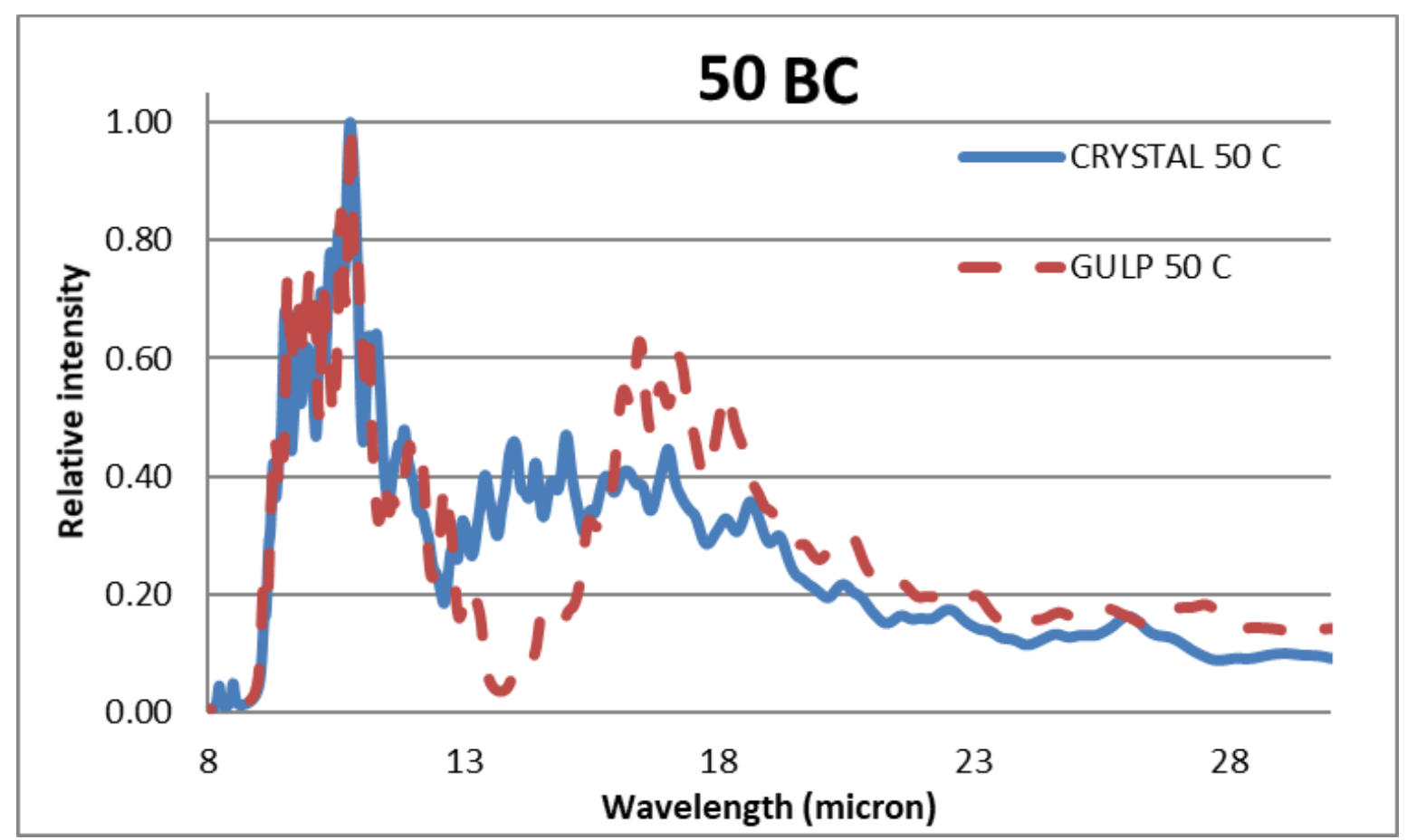

Figure S17 CRYSTAL vs GULP: IR spectra comparison for the 50 BC NP. 


\section{S5.3 GULP spectra}

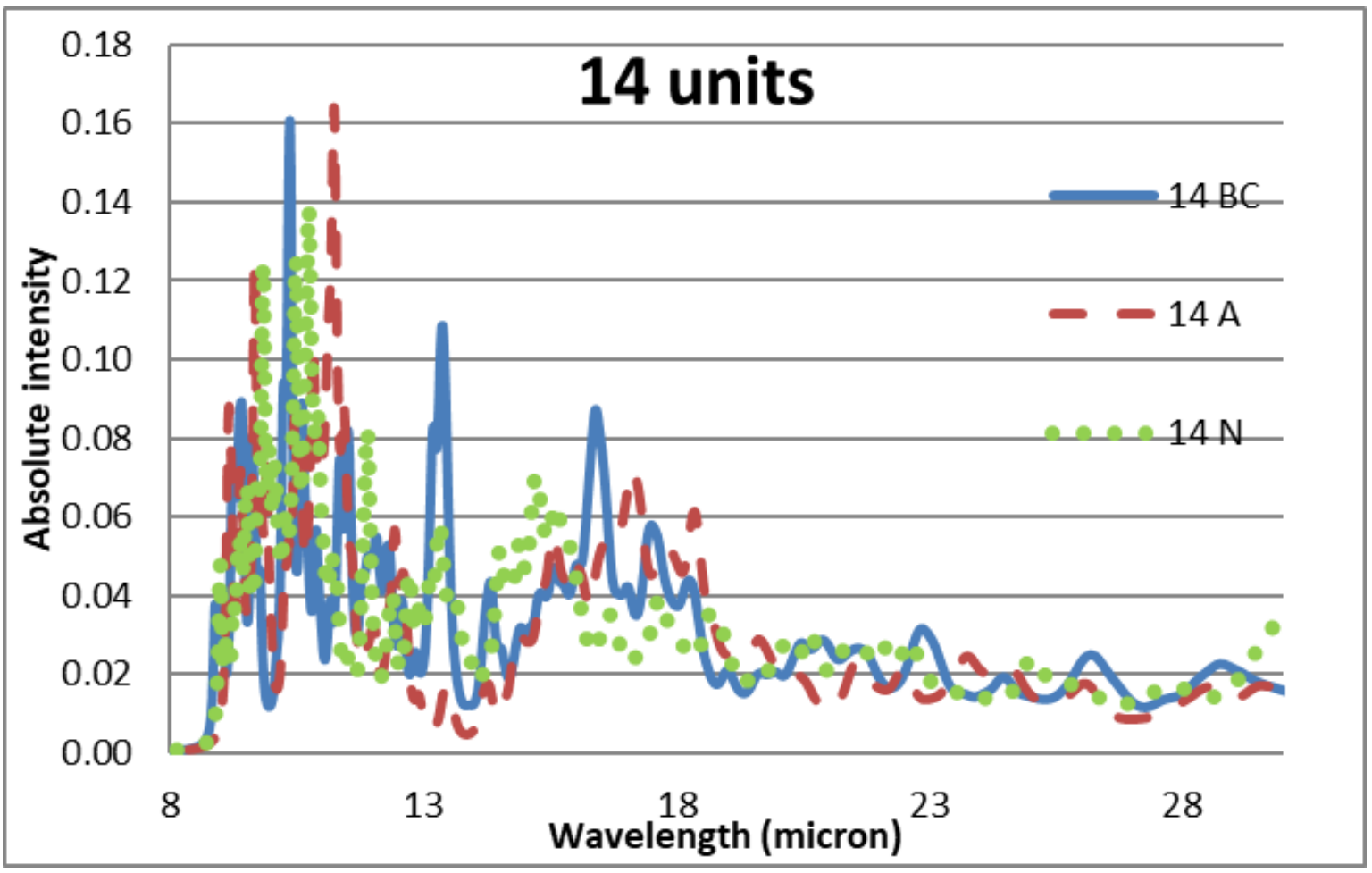

Figure S18 GULP simulated IR spectra for the 14 formula unit NPs.

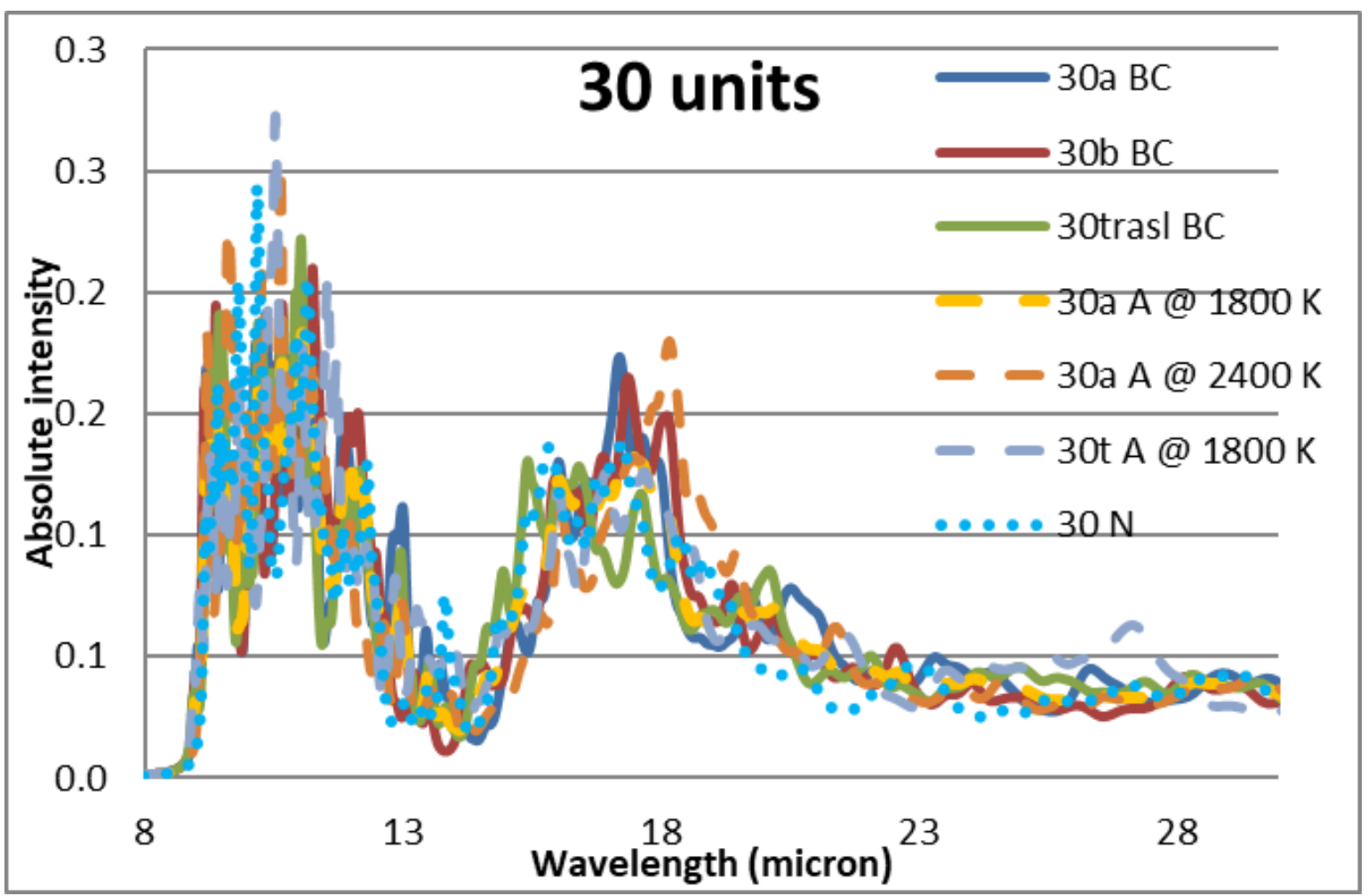

Figure S19 GULP simulated IR spectra for the 30 formula unit NPs. 


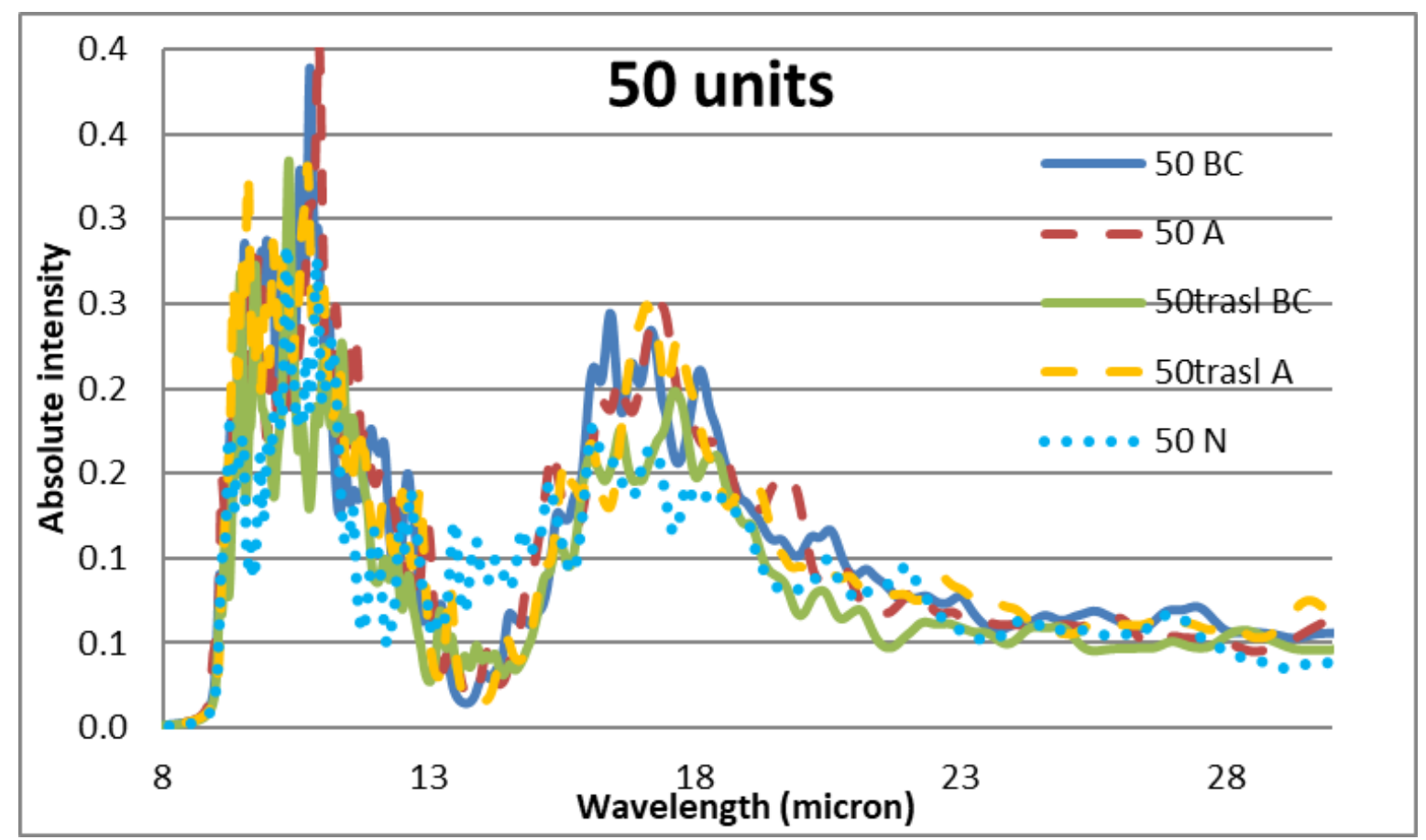

Figure S20 GULP simulated IR spectra for the 50 formula unit NPs.

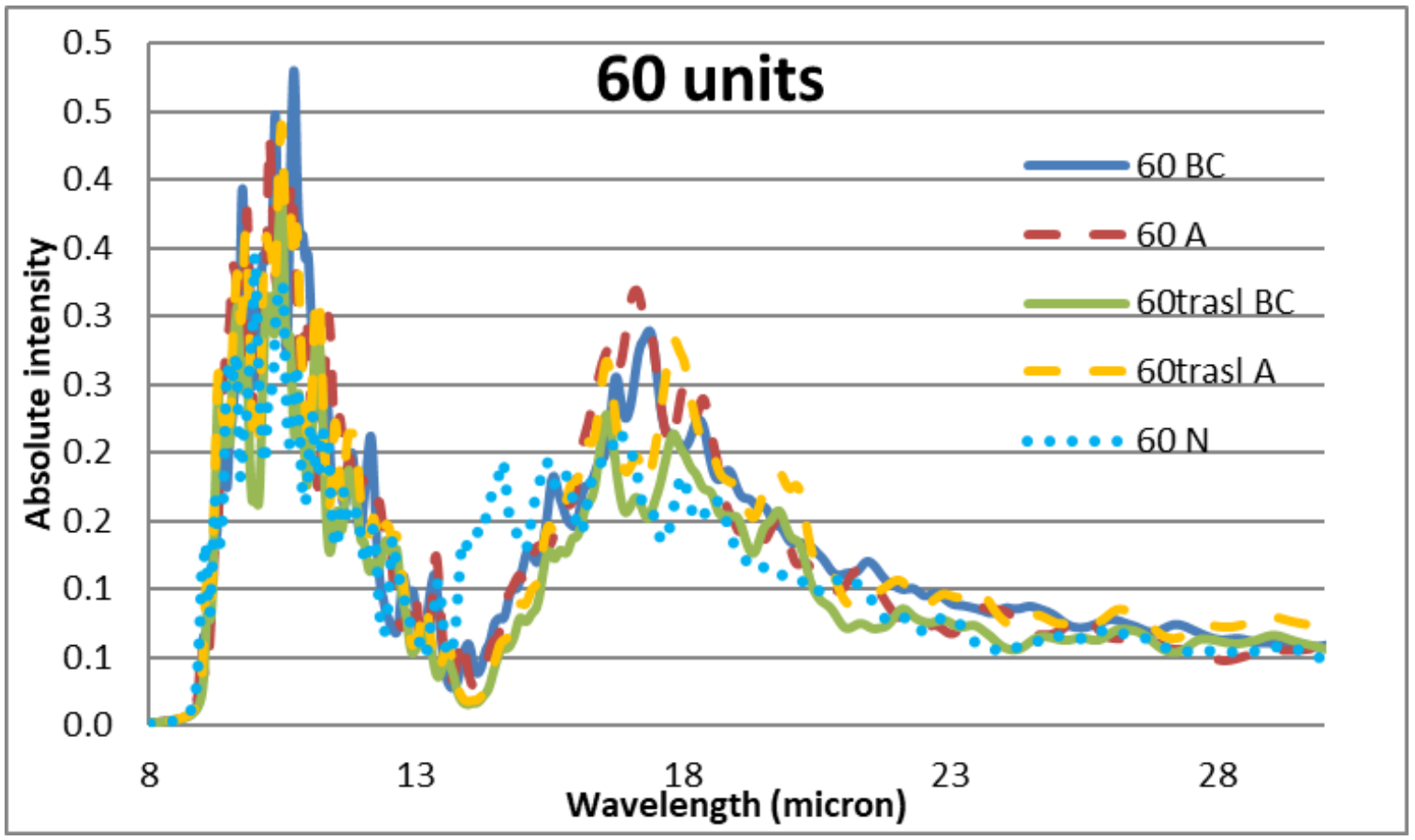

Figure S21 GULP simulated IR spectra for the 60 formula unit NPs. 


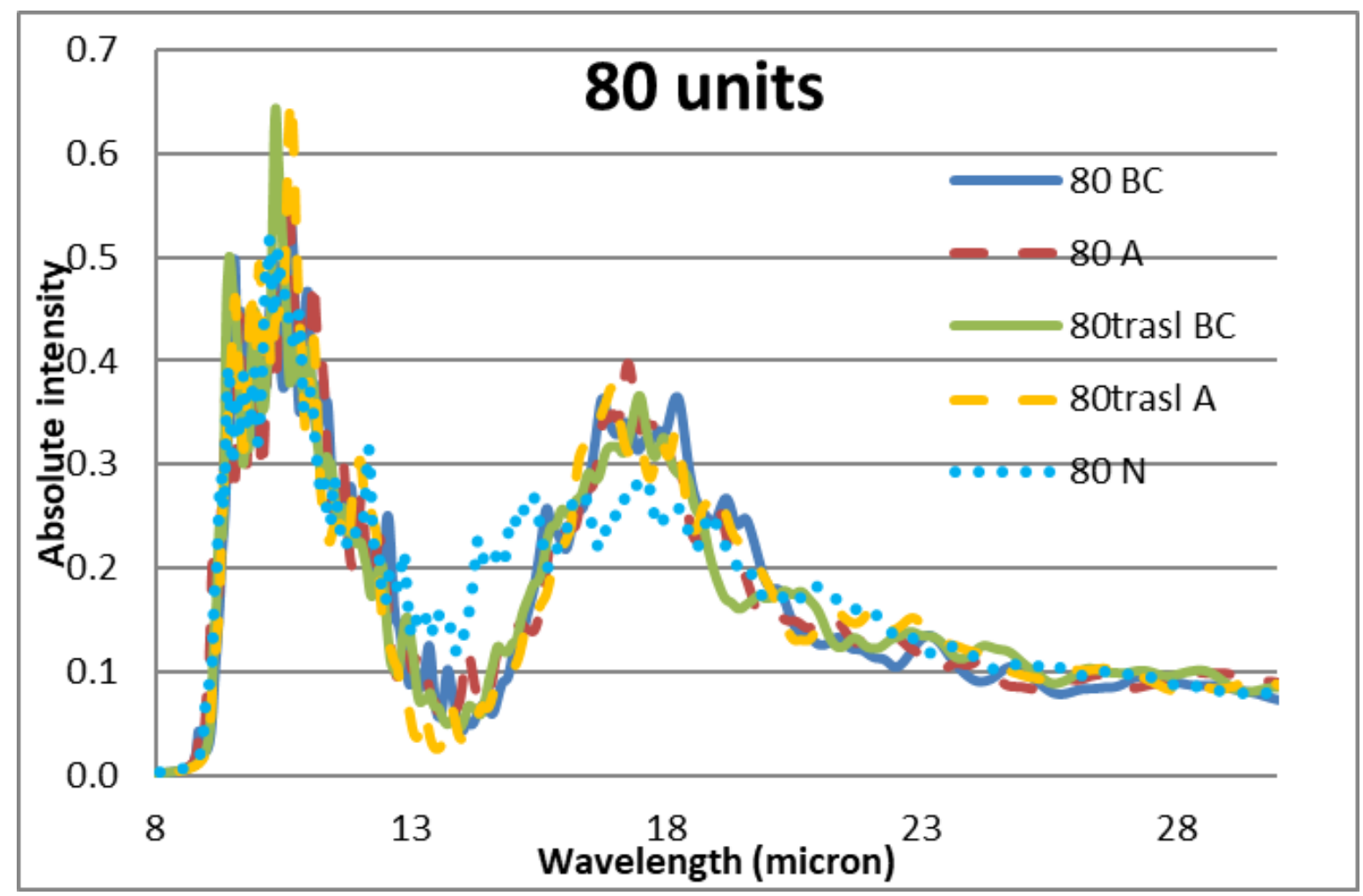

Figure S22 GULP simulated IR spectra for the 80 formula unit NPs.

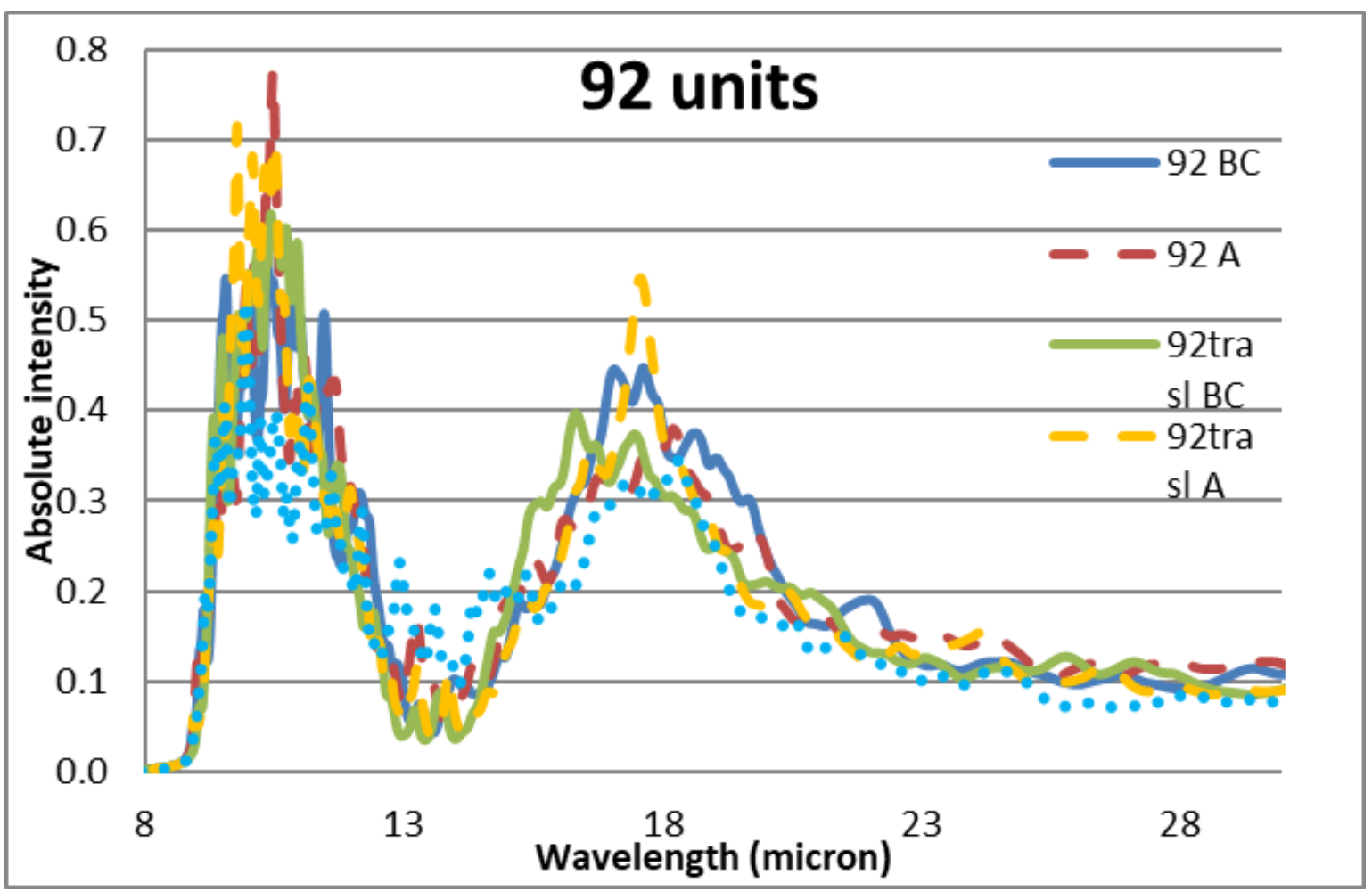

Figure S23 GULP simulated IR spectra for the 92 formula unit NPs. 


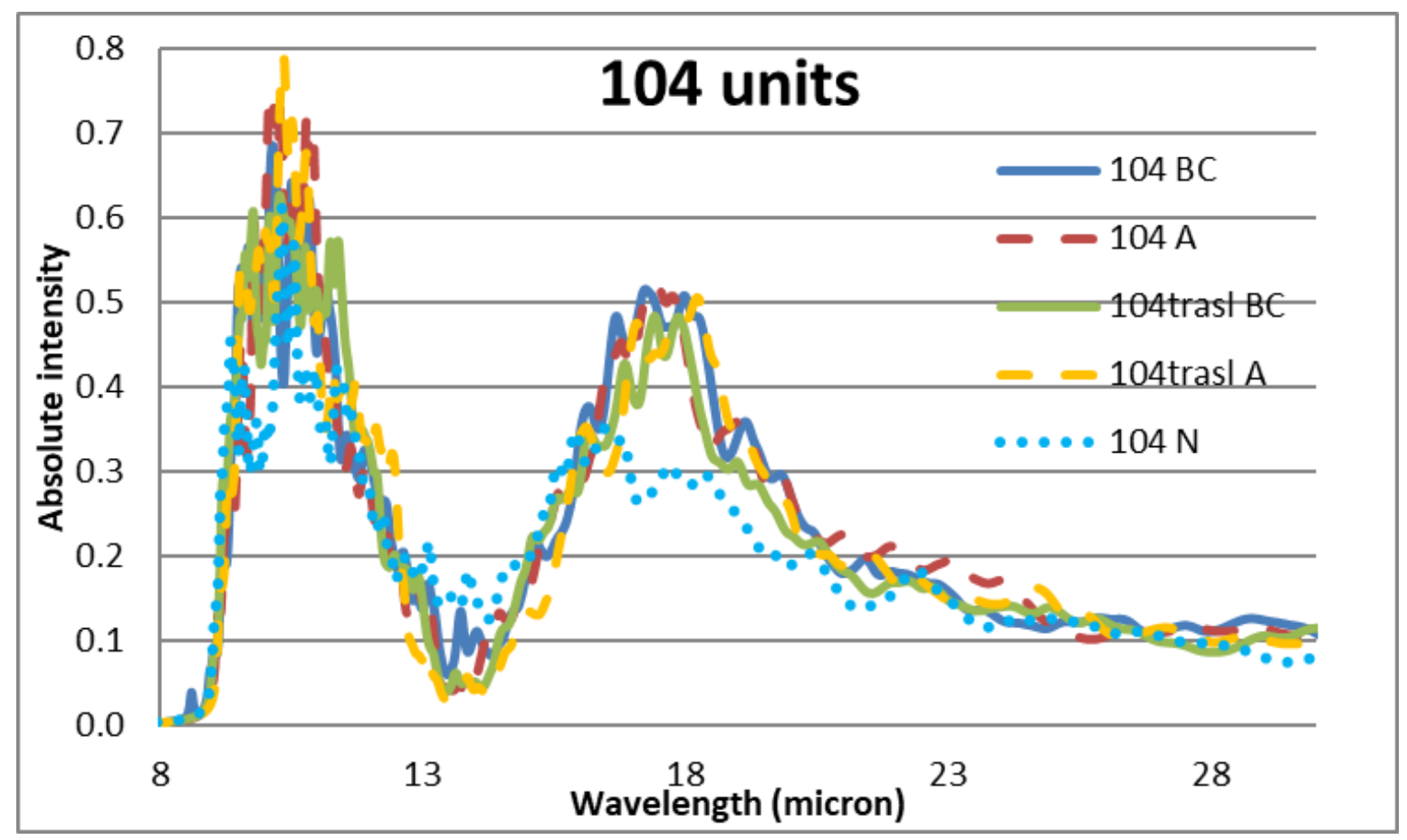

Figure S24 GULP simulated IR spectra for the 104 formula unit NPs.

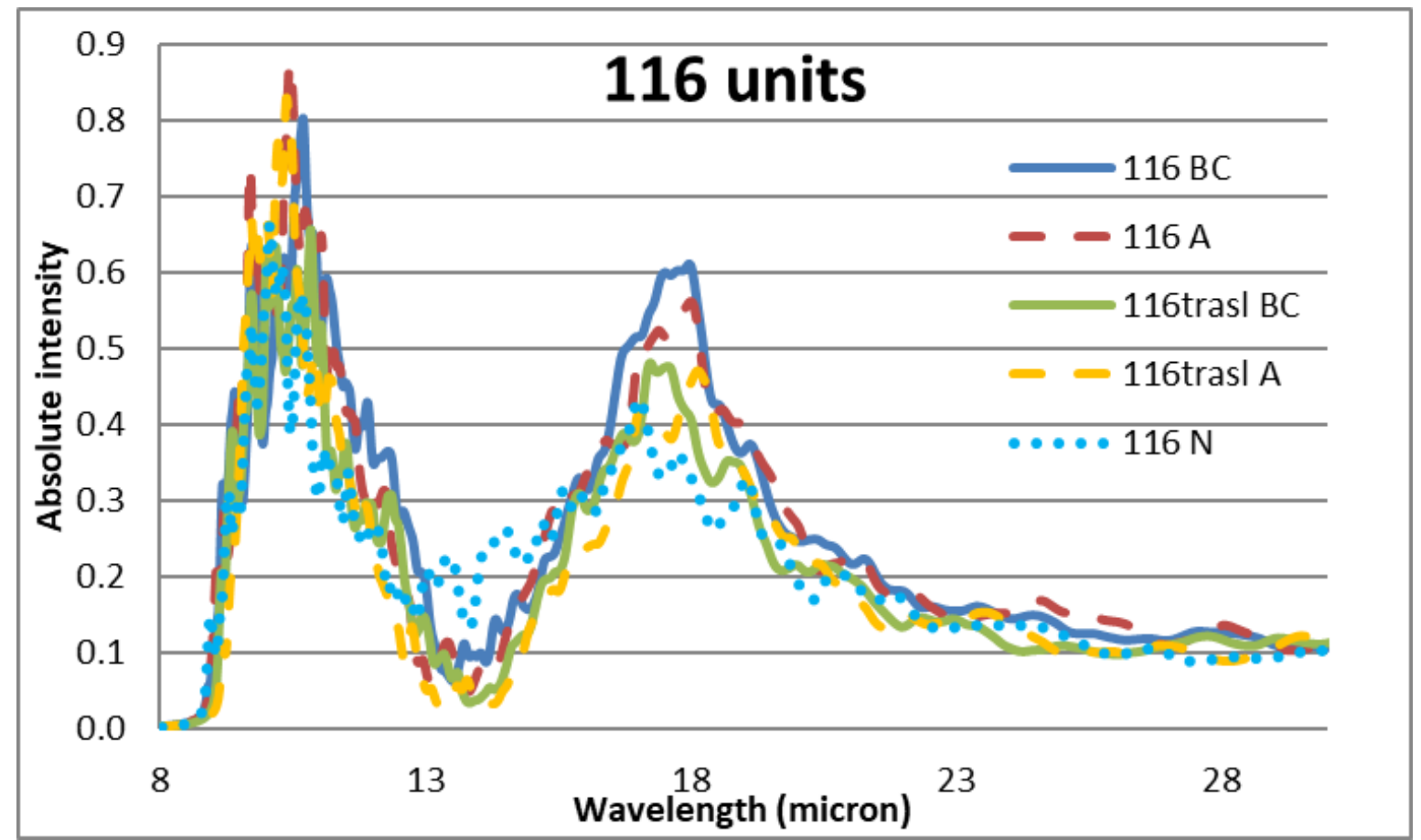

Figure S25 GULP simulated IR spectra for the 116 formula unit NPs. 


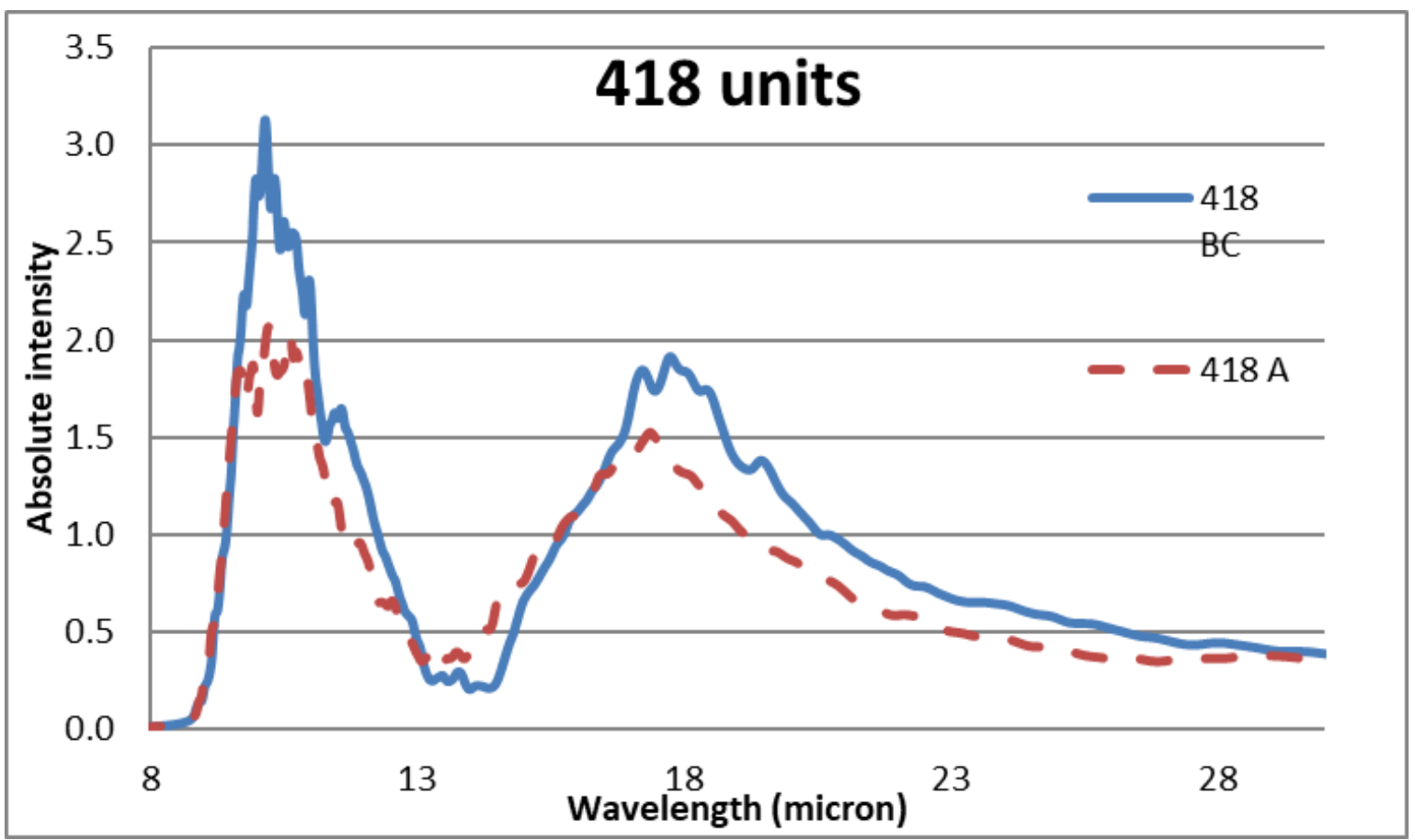

Figure S26 GULP simulated IR spectra for the 418 formula unit NPs.

S5 Si-O BOND AND O-Si-O ANGLE DISTRIBUTIONS FOR 116 FORMULA-UNITS NPs

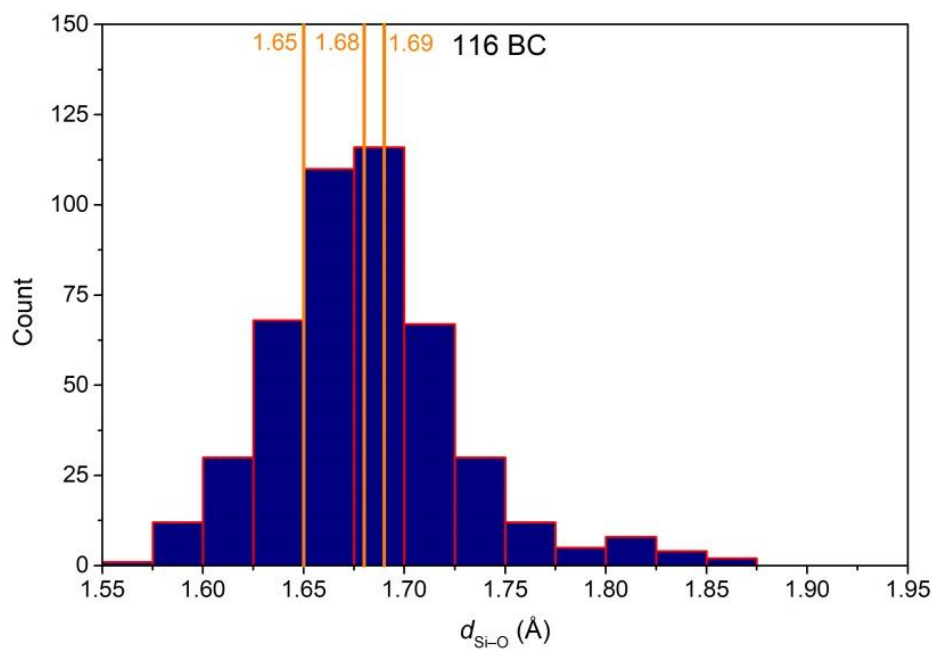

Figure S27 Si-O bond distance distribution for the 116 formula-units BC NP (vertical orange lines indicate values calculated for the bulk forsterite crystal). 


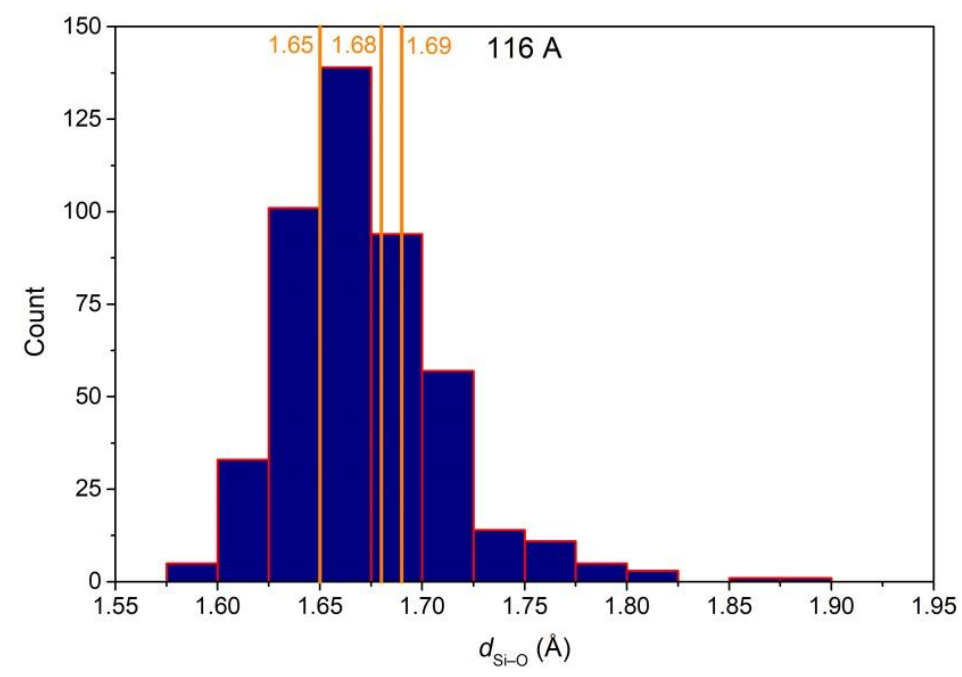

Figure S28 Si-O bond distance distribution for the 116 formula-units A NP (vertical orange lines indicate values calculated for the bulk Forsterite frystal).

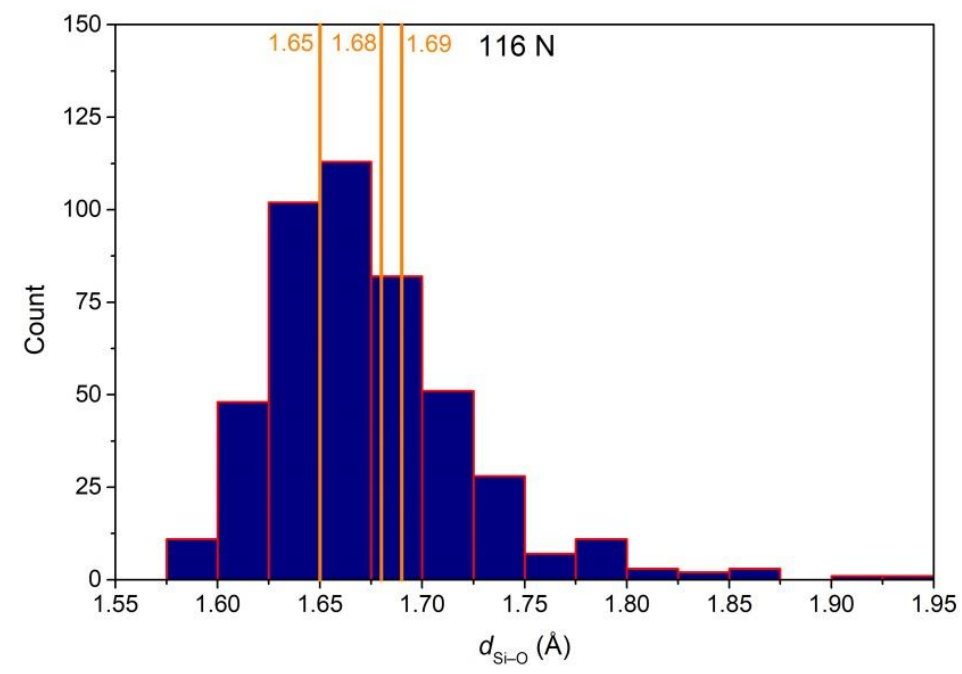

Figure S29 Si-O bond distance distribution for the 116 formula-units N NP (vertical orange lines indicate values calculated for the bulk forsterite crystal). 


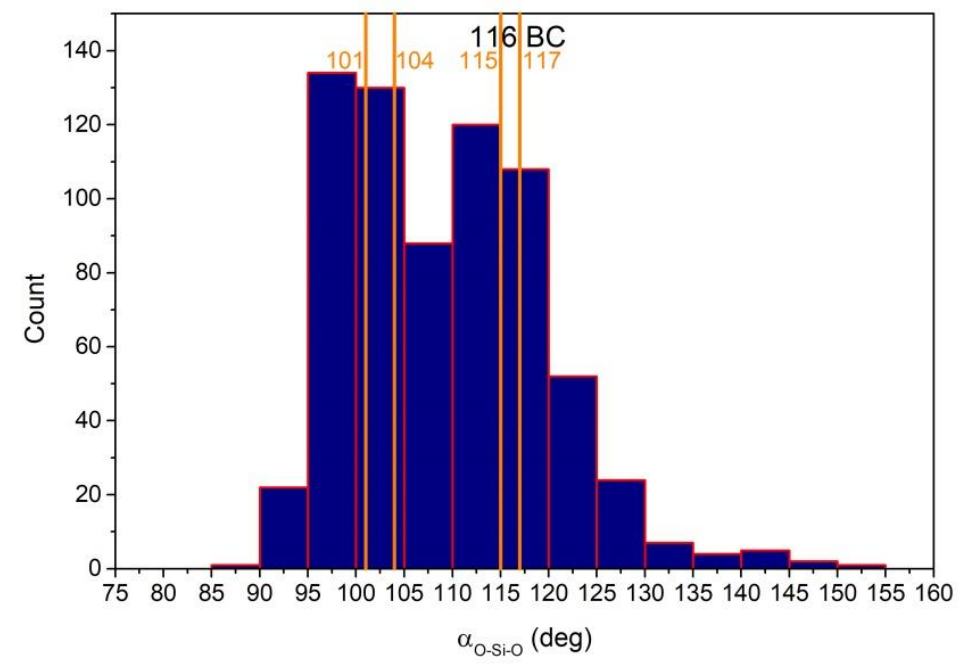

Figure S30 O-Si-O bond angle distribution for the 116 formula-units BC NP (vertical orange lines indicate values calculated for the bulk forsterite crystal).

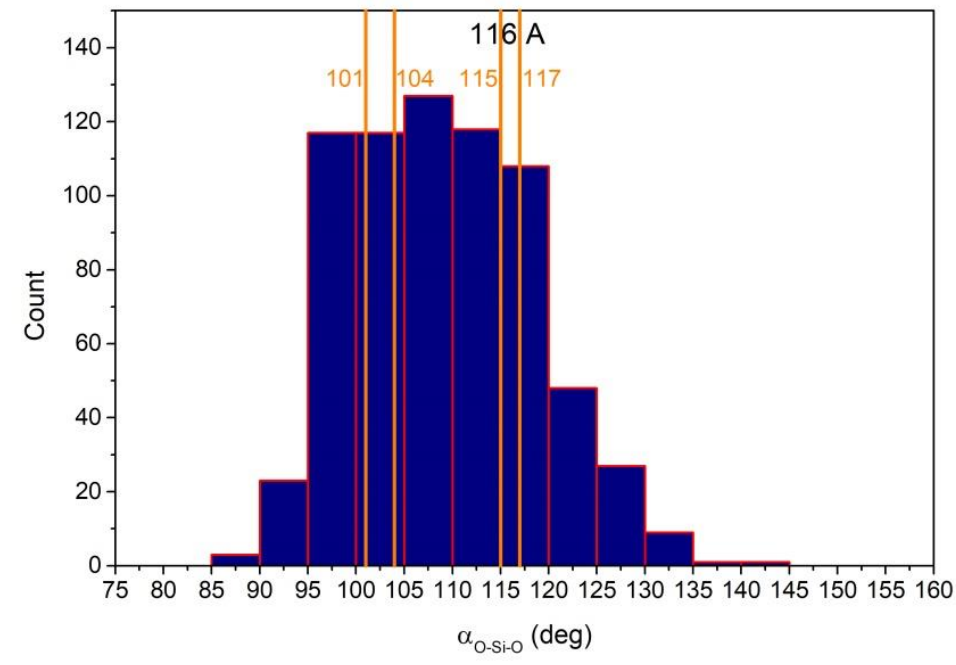

Figure S31 O-Si-O bond angle distribution for the 116 formula-units A NP (vertical orange lines indicate values calculated for the bulk forsterite crystal). 


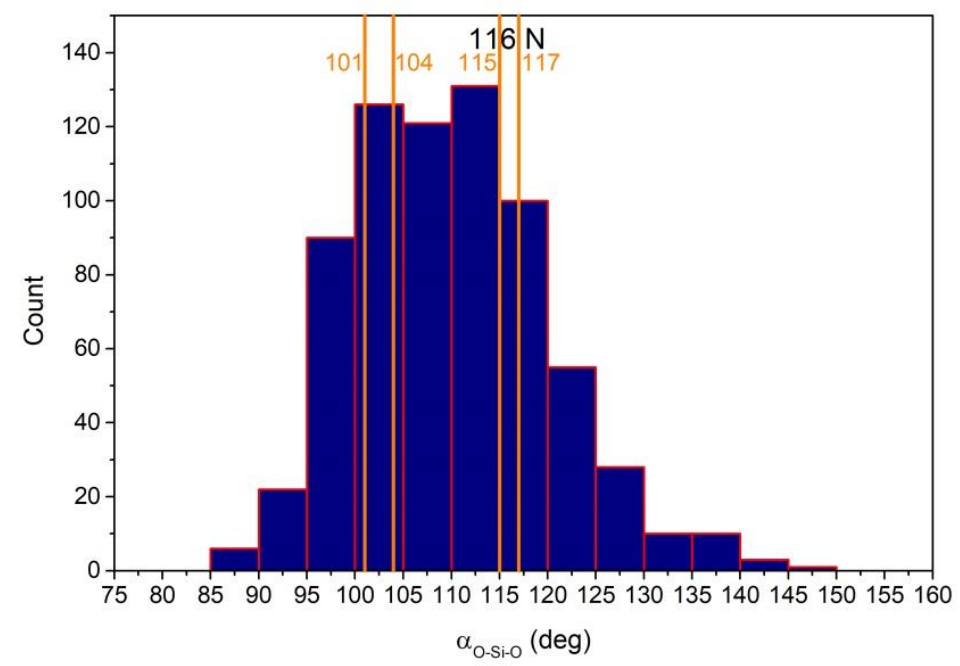

Figure S32 O-Si-O bond angle distribution for the 116 formula-units N NP (vertical orange lines indicate values calculated for the bulk forsterite crystal).

\section{REFERENCES}

(1) Noël, Y.; Zicovich-Wilson, C. M.; Civalleri, B.; D’Arco, P.; Dovesi, R. Polarization Properties of $\mathrm{ZnO}$ and $\mathrm{BeO}$ : An Ab Initio Study through the Berry Phase and Wannier Functions Approaches. Phys. Rev. B 2001, 65, 0141(11-20).

(2) Bostrom, D. Single-Crystal X-Ray Diffraction Studies of Synthetic Ni-Mg Olivine Solid Solution. Am. Mineral. 1987, 72, 965-972.

(3) Suto, H.; Sogawa, H.; Tachibana, S.; Koike, C.; Karoji, H.; Tsuchiyama, A.; Chihara, H.; Mizutani, K.; Akedo, J.; Ogiso, K.; et al. Low-Temperature Single Crystal Reflection Spectra of Forsterite. Mon. Not. R. Astron. Soc. 2006, 370, 1599-1606.

(4) Momma, K.; Izumi, F. VESTA 3 for Three-Dimensional Visualization of Crystal, Volumetric and Morphology Data. J. Appl. Crystallogr. 2011, 44, 1272-1276.

(5) Bruno, M.; Massaro, F. R.; Prencipe, M.; Demichelis, R.; De La Pierre, M.; Nestola, F. Ab Initio Calculations of the Main Crystal Surfaces of Forsterite $\left(\mathrm{Mg}_{2} \mathrm{SiO}_{4}\right)$ : A Preliminary Study to Understand the Nature of Geochemical Processes at the Olivine Interface. J. Phys. Chem. C 2014, $118,2498-2506$.

(6) Wulff, G. Zur Frage Der Geschwindigkeit Des Wachstums Und Der Auflosung Der Krystallflachen. Zeitschrift für Kryst. und Mineral. 1901, 34, 449-530.

(7) Zamirri, L.; Corno, M.; Rimola, A.; Ugliengo, P. Forsterite Surfaces as Models of Interstellar Core Dust Grains: Computational Study of Carbon Monoxide Adsorption. ACS Earth Sp. Chem. 2017, 1, 
384-398.

(8) Watson, G. W.; Oliver, P. M. Computer Simulation of the Structure and Stability of Forsterite Surfaces. Phys. Chem. Miner. 1997, 25, 70-78.

(9) de Leeuw, N. H.; Parker, S. C.; Catlow, C. R. A. Modelling the Effect of Water on the Surface Structure and Stability of Forsterite. Phys. Chem. Miner. 2000, 27, 332-341.

(10) Stewart, J. J. P. MOPAC2016. MOPAC2016, Stewart Computational Chemistry, http://openmopac.net. Colorado Springs, CO, USA 2016.

(11) Gale, J. D. GULP - A Computer Program for the Symmetry Adapted Simulation of Solids. J. Chem. Soc. Faraday Trans 1997, 93, 629.

(12) van Duin, A. C. T.; Dasgupta, S.; Lorant, F.; Goddard III, W. A. ReaxFF: A Reactive Force Field for Hydrocarbons. J. Phys. Chem. A 2001, 105, 9396-9409.

(13) Goumans, T. P. M.; Bromley, S. T. Efficient Nucleation of Stardust Silicates via Heteromolecular Homogeneous Condensation. Mon. Not. R. Astron. Soc. 2012, 420, 3344-3349.

(14) Plimpton, S. Fast Parallel Algorithms for Short-Range Molecular Dynamics. J. Comput. Phys. 1995, 117, 1-19.

(15) McQuarrie, D. A. Statistical Mechanics; University Science Books: Sausalito, CA, USA, 2000. 